\title{
1 Zebrafish Dscaml1 is Essential for Retinal Patterning and Function of
}

\section{Oculomotor Subcircuits}

4 Manxiu Ma1,2\#, Alexandro D. Ramirez ${ }^{3 \#}$, Tong Wang1\#, Rachel L. Roberts ${ }^{1,4}$, Katherine E. Harmon ${ }^{5}$, 5 David Schoppik ${ }^{5}$, Avirale Sharma1, Christopher Kuang1,6, Stephanie L. Goei7, James A. Gagnon ${ }^{8,9}$, 6 Steve Zimmerman ${ }^{8}$, Shengdar Q. Tsai10,11,12, Deepak Reyon 10,11,13, J. Keith Joung10,11, Emre R. F. $7 \quad$ Aksay $^{3}$, Alexander F. Schier 8,14 , and Y. Albert Pan ${ }^{1,2 *}$

$9{ }^{1}$ Department of Neuroscience and Regenerative Medicine, Medical College of Georgia, Augusta University, 10 Augusta, GA 30912.

$11{ }^{2}$ Center for Neurobiology Research, Fralin Biomedical Research Institute at Virginia Tech Carilion, Virginia 12 Tech, VA 24016.

13 3Institute for Computational Biomedicine and the Department of Physiology and Biophysics, Weill Cornell 14 Medical College, New York, New York 10021.

$15{ }^{4}$ Graduate Program in Neuroscience, Augusta University.

16 5Depts. of Otolaryngology, Neuroscience \& Physiology, and the Neuroscience Institute, New York University 17 Langone School of Medicine, New York, NY 10016.

18 6Medical Scholars Program, Augusta University.

19 7Department of Ophthalmology, Medical College of Georgia, Augusta University, Augusta, GA 30912

20 8Department of Molecular and Cellular Biology, Harvard Stem Cell Institute, Center for Brain Science, 21 Harvard University, Cambridge, MA 02138.

$22{ }^{9}$ Current address: School of Biological Sciences, University of Utah, Salt Lake City, Utah 84412

$23{ }^{10}$ Molecular Pathology Unit, Center for Computational and Integrative Biology, and Center for Cancer

24 Research, Massachusetts General Hospital, Charlestown, MA 02129.

25 11Department of Pathology, Harvard Medical School, Boston, MA 02115.

$26{ }^{12}$ Current address: Department of Hematology, St. Jude Children's Research Hospital, Memphis, TN, 38105. 
$27 \quad{ }^{13}$ Current address: Editas Medicine, 11 Hurley Street, Cambridge, MA 02142.

28 14The Broad Institute of Massachusetts Institute of Technology and Harvard, Cambridge, MA 02142.

29 \#T.W., M.M., and A.R. contributed equally to this article.

$31{ }^{*}$ Correspondence should be addressed to Y. Albert Pan, Center for Neurobiology Research, Fralin

32 Biomedical Research Institute at VTC, 2 Riverside Circle, Roanoke, VA 24016. E-mail:yapan@vtc.vt.edu.

33

34 Conflict of Interest: J.K.J. has financial interests in Beam Therapeutics, Editas Medicine, Pairwise Plants,

35 Poseida Therapeutics, Transposagen Biopharmaceuticals, and Verve Therapeutics. J.K.J.'s interests were

36 reviewed and are managed by Massachusetts General Hospital and Partners HealthCare in accordance with

37 their conflict of interest policies. J.K.J. holds equity in Excelsior Genomics. J.K.J. is a member of the Board of

38 Directors of the American Society of Gene and Cell Therapy. J.K.J. is a co-inventor on various patents and

39 patent applications that describe gene editing and epigenetic editing technologies. 


\section{Abstract}

42 Down $\underline{\text { Syndrome }}$ Cell $\underline{\text { Adhesion }}$ Molecules (dscam and dscaml1) are essential regulators of neural 43 circuit assembly, but their roles in vertebrate neural circuit function are still mostly unexplored. We 44 investigated the role of dscaml1 in the zebrafish oculomotor system, where behavior, circuit 45 function, and neuronal activity can be precisely quantified. Loss of zebrafish dscaml1 resulted in 46 deficits in retinal patterning and light adaptation, consistent with its known roles in mammals. 47 Oculomotor analyses showed that mutants have abnormal gaze stabilization, impaired fixation, 48 disconjugation, and faster fatigue. Notably, the saccade and fatigue phenotypes in dscaml1 mutants 49 are reminiscent of human ocular motor apraxia, for which no animal model exists. Two-photon 50 calcium imaging showed that loss of dscaml1 leads to impairment in the saccadic premotor pathway 51 but not the pretectum-vestibular premotor pathway, indicating a subcircuit requirement for 52 dscaml1. Together, we show that dscaml1 has both broad and specific roles in oculomotor circuit 53 function, providing a new animal model to investigate the development of premotor pathways and 54 their associated human ocular disorders.

\section{Introduction}

The Down Syndrome Cell Adhesion Molecule (DSCAM) family proteins are neuronal cell recognition molecules that are essential for neural circuit assembly and neural patterning across different phyla. In humans, loss of DSCAM and DSCAM-like 1 (DSCAML1) are linked to autism spectrum disorder and cortical abnormalities 1, 2. At the cellular level, DSCAMs promote avoidance between neurites of the same cell or between similar cell types. DSCAMs are also involved in the regulation of cell death,

62 synaptic adhesion, axon outgrowth, axonal refinement, and dendritic growth 3, 4, 5, 6, 7, 8 . However, it 63 is still unclear how the sum of these cellular functions contribute to neural circuit activity, behavior, and human disorders.

Here, we investigate the role of DSCAML1 on neural circuit function in the context of oculomotor 66 behavior, a setting with a rich history and easy to quantify behaviors. Eye movement is an integral 67 part of visual perception and survival in vertebrates, enabling shifts in visual attention and 
68 preventing image blurring during object or head motion. We focus on saccade, fixation, and gaze 69 stabilization movements, which are highly conserved among vertebrates. Saccades are fast ballistic 70 eye movements that allow rapid gaze shifting and redirection of visual attention. After each saccade, 71 gaze is maintained at a single location, termed fixation. Saccades also occur reflexively during gaze 72 stabilization, e.g., the optokinetic reflex (OKR) and vestibular ocular reflex (VOR) ${ }^{9}$. OKR is triggered 73 by broad-field visual motion, during which the eyes counteract motion by velocity-matched eye 74 movements. The periods of velocity matching, called 'slow phase', are interrupted by 'fast phase' 75 saccadic movements to the opposite direction, which reset eye position. Slow and fast phases are also triggered by the vestibular system in the VOR to counteract head motion.

77 In the oculomotor system, the neural circuitry is mapped in detail, and changes to eye movement 78 kinematics can be attributed to specific subcircuit deficits. Sensory inputs and motor commands 79 from higher brain centers converge onto the brainstem, where premotor neurons generate motor 80 signals encoding eye position and velocity. The premotor pathways, in turn, activate the cranial 81 motor neurons that innervate the extraocular muscles (Fig. 1A). Three subcircuits are the focus of 82 this study. The saccadic premotor pathway, which goes through the excitatory burst neurons in the 83 midbrain and hindbrain, initiates saccade and encodes the appropriate eye velocity. The vestibular 84 premotor pathway, which receives input from the eye and ear, controls slower eye movement and 85 velocity matching during gaze stabilization. Lastly, the neural integrator premotor pathway 86 integrates information from the saccade and vestibular pathways to maintain stable eye position 87 over time. Deficits in these subcircuits are associated with human diseases such as ocular motor 88 apraxia (saccade deficit), optokinetic response abnormalities (vestibular deficit), and gaze-evoked 89 nystagmus (neural integrator deficit) ${ }^{9}$. Eye movement deficits are common in the general 90 population and can lead to severely impaired visual function and difficulty in daily tasks such as 91 reading or crossing the street. Saccade deficits are also highly prevalent in neuropsychiatric and 92 neurodegenerative patient populations, serving as a diagnostic tool and a window into the 93 underlying pathophysiology 10,11. However, our understanding of the genetic contributors for 94 oculomotor circuit development and neural mechanisms for eye movement disorders are still 95 limited. 
96 We take advantage of the zebrafish oculomotor system as a framework to probe the role of $d s c a m l 1$, 97 the zebrafish homolog of DSCAML1, in neural circuit development, neuronal activity, and behavior

98 12. Zebrafish oculomotor behavior is robust at the larval stage; the small size and optically 99 translucency of zebrafish larvae make it possible to use two-photon calcium imaging to record 100 neuronal activity en masse while the animal is behaving ${ }^{13}$. Additionally, the ontogeny and anatomy 101 of the fish oculomotor system is well characterized and conserved with that of mammals 14,15 . We 102 found that genetic deletion of zebrafish dscaml1 affected retinal patterning, light adaptation, and 103 oculomotor behaviors. Our results suggest that for horizontal eye movements, the saccade and 104 integrator subcircuits are affected in dscaml1 mutants, while the vestibular subcircuit is mostly 105 spared. dscaml1 also affects the robustness of OKR against prolonged visual stimulation. The mutant 106 phenotype mirrors several characteristic features of congenital ocular motor apraxia (COMA) 107 (OMIM 257550) and provides insight into the potential neural mechanisms of COMA and other 108 human oculomotor disorders $16,17$.

\section{Results}

110 To investigate the role of dscaml1 in visual circuit activity and function, we first examined its 111 expression in zebrafish. Then we assessed how the loss of dscaml1 affected visual pathway 112 development and locomotor behavior. Finally, we analyzed oculomotor behavior and neural activity 113 to deduce the underlying neural circuit changes in dscaml1 mutants.

\section{Expression and gene targeting of zebrafish dscaml1}

115 We examined the expression of dscaml1 with whole-mount fluorescent in situ hybridization, from 116 the embryonic stage to five days post-fertilization (dpf), during which visually guided eye 117 movements begin to mature 18, 19. dscaml1 expression was enriched in the nervous system and 118 present in most brain regions (Fig. 1B-E). In the retina, dscaml1 was expressed in the inner nuclear 119 layer (INL, containing the amacrine, bipolar, and horizontal cells) and the ganglion cell layer (GCL, 120 containing retinal ganglion cells and amacrine cells) at $3 \mathrm{dpf}$ (Fig. 1F-G). At $5 \mathrm{dpf}$, dscaml1 121 expression was seen predominantly in the INL and sparsely in the GCL. These early and late 
122 expression patterns match the expression of mouse Dscaml1 in the retina at P6 and P12, 123 respectively $12,20$.

124 To test the function of dscaml1, we generated a mutant allele of $d s c a m l 1$ by TAL-like effector 125 nucleases (TALENs) mediated gene targeting ${ }^{21}$. The mutant allele, $d s c a m l 1^{\text {vt1 }}$, contains a seven base 126 pair deletion ( 9 base pair deletion plus 2 base pair insertion) after the start codon (Fig. $1 \mathrm{H})$. 127 Incorporation of the TALEN-mediated deletion in the dscaml1 mRNA was confirmed by RT-PCR and 128 sequencing. The deletion results in frame shift and a truncated open reading frame, which lacks the

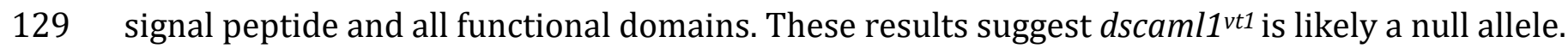

130 Homozygous mutant animals showed behavioral and morphological changes associated with the 131 visual system. At $5 \mathrm{dpf}$, homozygous mutant animals (dscaml1-/-) appeared to develop normally, 132 with normal head size, normal eye size, and inflated swim bladders. Compared with heterozygous 133 and wild-type siblings, however, mutant animals had darker pigmentation when placed on a light 134 background (Fig. 1I). This deficit in background adaptation, a camouflage response that requires 135 light detection, suggests that dscaml1-/- animals may be visually impaired 22. At later stages, 136 lethality was observed in mutant animals at $8 \mathrm{dpf}$, and none survived past $11 \mathrm{dpf}$ (Fig. 1J). 137 Heterozygous animals (dscaml1+/-) are viable and fertile as adults. Despite the late larval stage 138 lethality, dscaml1 mutant brain showed no gross abnormalities. Major longitudinal and 139 commissural axon tracts, motor axon terminals, and peripheral ganglia are formed normally in 140 mutants (Supplementary Fig. S1). The only observed morphometric difference was in the optic 141 tectum neuropil region, a major retinorecipient area important for sensory processing. The optic 142 tectum neuropil was 23\% larger in dscaml1 mutants $\left(44,121 \pm 1,017 \mu^{2}\right)$ versus wild type $143\left(35,816 \pm 656.5 \mu \mathrm{m}^{2}\right)$ or heterozygotes $\left(36,715 \pm 549 \mu \mathrm{m}^{2}\right) \quad(\mathrm{p}<0.0001$, one-way ANOVA) 144 (Supplementary Fig. S1D).

\section{5 dscaml1 is required for planar and laminar patterning in the retina}

146 Genetic studies in mice and chick demonstrated that Dscaml1 is required for planar patterning of 147 retinal amacrine cells and laminar specific neurite termination in the inner plexiform layer (IPL) ${ }^{6}$, 14820,23 . We found that dscaml1 has conserved functions in retinal patterning in zebrafish (Fig. 2). With 
H\&E staining, we did not see visible perturbation of retina structure, with the exception that the IPL

150 is thicker in the heterozygotes and mutants, compared to wild types (Fig 2A-B). Increased IPL 151 thickness was also seen in Dscam and Dscaml1 mutant mice, and may be caused by decreased 152 developmental cell death 20,23. To test dscaml1's role in planar patterning, we labeled the serotonin 153 (5-HT) expressing amacrine cells. 5-HT cells represent a single amacrine cell type (S1) that is 154 sparsely distributed in the retina, with very few contacts between cell bodies (Fig. 2C) ${ }^{24}$. In $d s c a m l 1$ 155 mutants, however, frequent clustering of 5-HT amacrine cells was observed. We calculated the 156 probability of one cell being immediately adjacent to another, termed aggregation index (density 157 recovery profile analysis was not possible due to the sparsity of this cell type in the larvae). 158 Compared to wild-type animals, the aggregation index in mutants was significantly increased (Fig. 159 2D). The increased aggregation seen in the mutants was not due to an increase in the number of 5160 HT amacrine cells, which was not significantly different between wild-type, heterozygote, and 161 mutant animals (Fig. 2E).

162 We also found that loss of dscaml1 impacted laminar specific neurite termination in the IPL, 163 specifically of the ON-bipolar cell axon terminals (visualized with anti-PKC $\alpha$ ) ${ }^{25}$. ON-bipolar cells 164 transduce electrical activity in response to light increments (e.g., lights turning on) and have 165 dendrites that extend toward the OPL (where they synapse with photoreceptor cells) and axons 166 that form three discrete layers in the inner half of the IPL (the ON sublamina) (Fig. 2F) ${ }^{26}$. This 167 distribution was quantified by measuring the fluorescent intensity of PKC $\alpha$ immunolabeling across 168 the thickness of the IPL, as defined by SV2 immunolabeling27. In all wild-type and heterozygote 169 animals, three distinct peaks at stereotypical positions can be discerned. In contrast, PKC $\alpha$ 170 distribution in the mutants was more diffuse and did not form three discrete sublaminae (Fig. 2G). 171 Cone photoreceptors and Müller glia appear morphologically normal in the dscaml1 mutant retina 172 (Fig. 3A-F).

173 Loss of dscaml1 did not appreciably affect the afferent projections of the retina. We utilized the $174 \mathrm{Tg}$ (atoh7:GAP-RFP) transgenic line, which expresses membrane-tagged red fluorescent protein in 175 retinal ganglion cells ${ }^{28}$. In dscaml1 mutants, the optic nerve crossed the chiasm normally, and the 176 axon terminals (arborization fields) of the optic tract were mostly indistinguishable from wild-type 
and heterozygous animals (Fig. 3G-I) ${ }^{29}$. This suggests that the expression of dscaml1 in the GCL is

178 not required for outgrowth of retinal ganglion cell axons. It remains possible, however, that the 179 projections of a subset of retinal ganglion cells may be affected by the loss of $d s c a m l 1$. Overall, the 180 expression and mutant phenotypes of zebrafish dscaml1 demonstrate that it is functionally 181 conserved with other vertebrates and required for both planar and laminar patterning in the retina.

\section{Abnormal locomotor behavior in dscaml1 mutants}

183 Given the retinal patterning deficits and background adaptation abnormality seen in the mutants, 184 we asked whether light-induced locomotor activity is also affected. We monitored locomotor 185 activity of individual $5 \mathrm{dpf}$ larvae over 24 hours, under normal circadian cycle (14 h day/10 h night) 186 (Fig. 4A) 30,31. Consistent with previous studies, wild-type fish were more active during the day, less 187 active at night, and responded robustly during day/night and night/day transitions ${ }^{30}$. This diurnal 188 rhythm was preserved in dscaml1 mutants. Loss of dscaml1 did not affect the duration of active 189 periods, during either day or night (Fig. 4B, C). The amount of movement, however, was reduced in 190 mutants during the day as well as the response to lights turning on, compared to wild-type and 191 heterozygous animals (Fig. 4D-F). Interestingly, the response in mutants to lights off was as robust 192 as for wild-type and heterozygous siblings (Fig. 4G). These results show that dscaml1 mutants can 193 detect light and move in response to a light offset, but are deficient in responding to light onset. 194 These behavioral deficits are consistent with a partial deficit in the retinal ON pathway, potentially 195 contributed by imprecise sublamina-specific ON-bipolar cell axon targeting.

\section{Abnormal OKR and eye lock up in dscaml1 mutants}

197 To further test the role of dscaml1 in neural circuit function and sensorimotor integration, we 198 examined the mutants' performance in OKR. OKR consists of visual motion (i.e., optic flow)199 triggered tracking eye movements (slow phases) and intervening resetting saccades (fast phases) 200 (Fig. 5A). The quality of OKR is measured by slow-phase gain, which is the ratio of eye velocity to 201 the velocity of the visual motion. In zebrafish, OKR develops early and is robust by $3-4 \mathrm{dpf} 18$. We 202 tested 5-6 dpf larvae inside a circular arena where black and white moving gratings were projected 203 onto the arena, and eye position was video-recorded simultaneously (Fig. 5A, Movie 1). Grating 
204 directions alternated between clockwise and counter-clockwise at 3 or 40 seconds (Fig. 5B, E).

205 Under short time scale (3 s), fast phase has little effect on OKR performance, and gain is directly 206 related to the processing of optic flow via the vestibular premotor pathway (Fig. 1A) 32, 33. Under 207 long time scale (40 s), saccades are necessary to reset eye position periodically, and the oculomotor 208 system needs to be robust against eye position drifts and fatigue in optic flow response 19, 34, 35, 36.

209 We found that visuomotor processing for optic flow is intact in dscaml1 mutants. Under short time 210 scale, both control (wild type and heterozygote) and mutant animals exhibited qualitatively normal 211 optokinetic responses (Fig. 5B-D). Slow-phase gain increased linearly with the logarithm of stimulus 212 contrast in both groups, and there was no difference in gain at lower contrast levels (contrast $\leq 0.2$ ) 213 (Fig. 5H). Mutants do have lower slow-phase gain than the controls at higher contrast levels 214 (contrast=0.5 or 1), though the deficit is mild. These findings suggest that dscaml1 is likely not 215 required for the assembly or function of the vestibular premotor pathway, but affects OKR performance at conditions that elicit higher eye velocity ${ }^{32}$.

217 Under long time scale, dscaml1 mutants showed deficits in saccadic eye movements and exhibited 218 more pronounced behavioral fatigue. In control animals, fast phase (resetting saccade) and slow 219 phase alternated regularly, and saccades had consistent frequency and amplitude (Fig. 5F). In 220 mutants, resetting saccades were irregular and reduced in amplitude (Fig. 5G, I). Interestingly, 221 mutant eye movements would frequently pause, similar to the "lock up" phenotype seen in human 222 with saccade deficits ${ }^{16}$. Lock-up periods tended to occur towards the end of the stimulus period, 223 and the probability of lock up increased linearly over time, both in controls and mutants (Fig. 5J). 224 The probability of lock up (time bins where velocity $<1^{\circ} / \mathrm{s}$ ) was significantly higher in mutants, 225 compared to controls (comparison of linear regression intercepts, $p<0.0001$ ) ${ }^{35,36}$. Lock-up events 226 could occur throughout the eye position range in the mutants, suggesting that lock up is not due to 227 a failure to initiate faste phase at more eccentric positions (Supplementary Figure S2). Concurrently, 228 relative to controls, mutants had more pronounced decay of slow-phase gain over time, even when 229 lock-up periods were excluded (Fig. 5K, L) 19, 37 . In both control and mutant data sets, the decay of 230 slow-phase gain was well fit with a double exponential. Loss of dscaml1 resulted in a drop in the 231 contribution by the longer (later) component, and faster decay in the shorter (earlier) component 
232 (Table I., decay constant for short component shown in Fig. 5K, L). These results suggest that

233 dscaml1 has important roles in saccade generation and velocity maintenance.

234 dscaml1 is required for torsional eye movements during VOR

235 Our OKR assays tested the capacity for horizontal gaze stabilization, which requires the lateral and 236 medial recti. To test the functionality of the other four extraocular motoneuron populations, we 237 measured the gravito-inertial VOR in the torsional plane (Fig. 5M, N). In response to nose-up pitch 238 tilts, larvae will use their superior oblique and inferior rectus muscles to counter-rotate the eyes. 239 Similarly, following nose-down pitch tilts, larvae stabilize their gaze using the superior rectus and 240 inferior oblique muscles ${ }^{38}$. As expected, wild-type larvae at $5 \mathrm{dpf}$ showed strong VOR in response to $24115^{\circ}$ steps up or down away from horizontal, with a slightly stronger response to nose-up tilts.

242 dscaml1 mutants were able to initiate VOR in a directionally appropriate manner but had lower gain 243 (max eye velocity/max table velocity, 35\% $/$ ) to both stimuli. As fish develop VOR in the absence of 244 light, this deficit is likely not due to any visual impairment ${ }^{18}$. We conclude that dscaml1 is involved 245 in the performance of both the OKR and VOR, and in both the horizontal and torsional planes.

\section{6 dscaml1 is required for saccade and fixation}

247 Given the pronounced reduction in fast-phase response and faster decay of slow-phase response 248 during OKR, we next examined spontaneous eye movements in the absence of structured visual or 249 vestibular stimulus to assess specific deficits in saccade generation and neural integrator function. 250 Spontaneous eye movements in zebrafish consist of intermittent saccades, followed by periods of 251 fixation, similar to mammalian scanning saccades 19, 34. This behavior is used to direct visual 252 attention to the temporal retina, where photoreceptor density is highest 39 , 40. Spontaneous saccades 253 are conjugated and usually alternate in direction, with typical angular velocity (sampled at $5 \mathrm{~Hz}$ ) 254 greater than $100^{\circ}$ s (Fig. 6A). In control animals, spontaneous saccades with velocity greater than $255100^{\circ} / \mathrm{s}$ were the predominant type of eye movement (59\%) (Fig. 6C, D). In mutant animals, high256 velocity saccades were nearly absent (Fig. 6B-D). Instead of alternating in directions, sequential 257 saccades often moved in the same direction, suggesting that these eye movements may be short of 
258 their intended target (hypometric). Saccades in mutants were also significantly more disconjugated, 259 indicating that saccade initiation was bilaterally desynchronized (Fig. 6B, E).

260 The reduced saccade velocity in mutants may reflect slower eye movements caused by dysfunction 261 in the oculomotor periphery. Alternatively, reduced saccade velocity may be coincidental to smaller 262 saccade amplitudes, which generally have lower velocity. We tested the correlation of peak velocity 263 and saccade amplitude, a linear relationship known as main sequence 19, 41. Main sequence is a 264 clinically relevant diagnostic metric for saccade: patients with COMA have smaller saccade but 265 normal velocity (normal main sequence), whereas patients with saccade deficits associated with 266 neurodegenerative disorders have both smaller saccade and lower velocity (lower main sequence) 267 17, 42. We tested the spontaneous saccade main sequence (sampled at $30 \mathrm{~Hz}$ ) and found that there 268 was no significant difference between mutants and controls (p=0.468, ANCOVA) (Fig. 6F). In other 269 words, saccades in dscaml1 had smaller amplitude, but velocity was normal for the given amplitude. 270 The normal main sequence in mutants also suggests that the ocular periphery is not significantly 271 affected by the loss of dscaml1. Consistent with this finding, we did not see any gross abnormality 272 in the abducens motor neuron projection to the lateral rectus muscle in the mutants (Fig. 6G, H).

273 In addition to saccade deficits, post-saccade fixation was significantly perturbed in $d s c a m l 1$ 274 mutants, indicating a deficit in the neural integrator pathway. The integrator pathway provides 275 tonic activation of motor neurons to maintain fixation, counteracting spring forces in the eye plant 276 that would cause a drift back to the null position (arrows in Fig. 6A, B); during sustained OKR in a 277 given direction, this pathway is also necessary for converting tonic eye velocity commands to ramp278 like eye position commands. We modeled this drift rate using an exponential decay function and 279 found that eye position in mutant animals drifted towards baseline more rapidly than in controls 280 (median $\tau=24$ and 7 seconds for control and mutant animals, respectively, Fig. 6I). Together, these 281 results suggest that $d s c a m l 1$ is involved in the function of the saccadic and integrator pathways, but 282 does not affect the function of the oculomotor periphery ${ }^{43}$.

\section{Loss of dscaml1 leads to neural activity deficits during OKR fast phase}


284 To understand the neurophysiological basis of the oculomotor phenotypes, we focused on the 285 activity of the abducens motor complex (ABD), which controls the extraocular muscles for 286 horizontal eye movements and serves as the convergence point for different premotor inputs (Fig. $2871 \mathrm{~A})^{44,45}$. We performed two-photon calcium imaging in control (dscaml1+/-) and mutant (dscaml1288 /-) fish in elavl3:H2B-GCaMP6f transgenic background while they performed OKR ${ }^{46}$. Eye positions 289 were recorded simultaneously with an infrared video camera and oculomotor behavior-encoding 290 ABD neurons (motor or internuclear) were then identified based on anatomical location and 291 calcium activity (Fig. 7A-B) ${ }^{13}$. To induce a mixture of optokinetic and spontaneous responses we 292 projected a grating stimulus in front of the animal and then moved that stimulus in a repeating 293 temporal pattern (Fig. 7C-D). We measured mean fluorescence following the onset of stimulus 294 movement in either the ipsilateral (e.g., clockwise for right ABD) or contralateral (e.g., 295 counterclockwise for right ABD) direction. Slow phase ABD activity (a proxy for vestibular pathway 296 activity) was calculated with deconvolved calcium signal for the first three seconds of the ipsilateral 297 or contralateral phases. Fast-phase ABD activity (a proxy for saccadic pathway activity) was calculated with deconvolved average fluorescence following saccadic eye movements in both directions. Since ABD neuron responses are direction selective, we could excite neurons in both hemispheres by alternating the direction of stimulus movement.

Loss of dscaml1 did not affect ABD activity during slow phase ( $\mathrm{n}=86$ cells from 3 control animals; $\mathrm{n}=424$ cells from 6 mutant animals). Population-average activity in the ABD was comparable in mutant and control animals following the onset of stimulus movement in the ipsilateral direction (Fig. 7E, F). In the contralateral direction, ABD population-average activity reached similar steadystate levels in mutant and controls. This result is consistent with dscaml1's relatively mild effect on slow phase at short time scale and suggests that the vestibular pathway is unaffected.

In contrast, $\mathrm{ABD}$ activity was substantially reduced in mutants during fast phase, relative to controls. The saccade-triggered average activity (STA) of ABD cells in the control animals were characterized by a rapid increase or decrease in deconvolved fluorescence following an ipsilateral or contralateral saccade, respectively (Fig. 7G, H). The STA of mutant ABD cells following fast-phase events in either direction was reduced in both directions, compared to controls. Comparing the distribution of trial-averaged STA amplitudes, dscaml1 mutants had significantly lower amplitudes 
313 than controls ( $\mathrm{p}<0.05$, two-sample K-S test, $\mathrm{n}=110$ cells from 3 control animals, $\mathrm{n}=253$ cells from 7 314 mutant animals).

315 Together, our results show that oculomotor deficits originate centrally and that dscaml1 affects ABD

316 response selectively during the fast phase. The population-level calcium response in ABD suggests 317 that mutant $\mathrm{ABD}$ neurons have normal ramping during the slow phase but lack the saccade318 associate burst activity seen in controls. These findings support the idea that dscaml1 plays a 319 specific role in the function of the saccadic pathway.

Discussion

321 We investigated the cellular and behavioral roles of $d s c a m l 1$ in zebrafish and took advantage of the 322 oculomotor system to deduce dscaml1's function within a defined neural circuit. Our results 323 underscore the importance of DSCAM proteins in retinal patterning and visuomotor function. Our 324 neurophysiological findings further showed that loss of dscaml1 leads to impairment in the saccadic 325 but not the pretectum-vestibular premotor pathway, indicating a subcircuit requirement for 326 dscaml1. The collection of oculomotor deficits in dscaml1 mutants bears a striking resemblance to 327 human COMA, for which no animal models exist.

329 Our results show that zebrafish dscaml1, like its mammalian ortholog, is required for maintaining 330 cellular spacing and refining neurites into discrete synaptic laminae in the retina. In the retina, 331 distinct cell types are organized in a mosaic pattern horizontally, and neurites stratify vertically in 332 precise layers in the inner and outer plexiform layers ${ }^{47}$. Both aspects of spatial patterning require 333 the function of DSCAMs. We show that in zebrafish, loss of dscaml1 causes serotonergic amacrine 334 cells to aggregate. In the IPL, axon terminals of PKC $\alpha$-positive ON-bipolar cells are more diffuse in 335 dscaml1 mutants, which likely affects laminar specific synaptogenesis. As ON-bipolar cell axon 336 maturation is activity independent, this diffuse terminal morphology reflects a delay or failure in 337 axon terminal development 26, 27. Interestingly, Dscam rather than Dscaml1 is involved in ON338 bipolar cell axon stratification in mice. Nevertheless, Dscaml1 is involved in the refinement of 339 neurite stratification in both mice (VGLUT3+ amacrine cells) and chicken (retinal ganglion cells) ${ }^{6}$, 
34023 . These results demonstrate the remarkable functional conservation of Dscaml1 across vertebrate 341 species.

342 How the retinal patterning deficits observed in dscaml1 mutants affect visual function in dscaml1 343 still remains to be tested, but the collection of behavioral phenotypes provides some clues. The 344 reduced light-on locomotor response and darker pigmentation in dscaml1 mutants are suggestive 345 of a reduction in ON pathway function. Consistent with this, the sluggish locomotor response to 346 light-on observed in the dscaml1 mutant animals resembles the no optokinetic response $c$ (nrc) 347 mutant, which completely lacks retinal ON-responses ${ }^{48}$. The more diffuse targeting of ON-bipolar 348 cell axons in dscaml1 mutants may lead to reduced strength or specificity of light-on responses. 349 However, in contrast to nrc animals, which cannot perform OKR, dscaml1 animals can still perform 350 OKR. Therefore, it is most likely that ON-response is reduced but not completely abolished in 351 dscaml1 mutants.

353 The combination of behavioral assays and functional imaging showed that dscaml1 was necessary 354 for the function of the oculomotor circuit. We focused our analysis to three subcircuits for horizontal 355 eye movements: the saccadic, vestibular, and integrator premotor pathways.

356 Loss of dscaml1 strongly affected the saccadic pathway, as deficits were observed in mutants during 357 both reflexive saccades (OKR) and spontaneous scanning saccades. This is consistent with 358 physiological observations showing that saccade-related activity in the abducens was significantly 359 decreased in mutants compared to controls. Retinal defects may contribute to the saccade deficits 360 observed in mutants, at least during reflexive saccades, but would not account for the full extent of 361 the phenotype. Instead, our results favor the hypothesis that abnormal function of the saccadic 362 premotor pathway is the primary cause of the saccade phenotype in dscaml1 mutants. It is 363 hypothesized that the saccade generator circuit relies on strong local recurrent feedback to 364 generate large and coordinated pulses ${ }^{49}$. This feedback may be easily disrupted through the 365 expected connectivity deficits induced by dscaml1 mutation. Another factor contributing to the 366 saccadic deficit could be attenuated connectivity at the EBN-ABD synapse. However, the 
367 disconjugation of saccades observed in dscaml1 mutants suggests that the pulse signal from the 368 saccade generators is already fragmented or weakened before being projected to the ABD.

369 We saw relatively mild deficits in the vestibular pathway. OKR slow phase performance at short 370 time scale was mostly normal in mutants, consistent with the normal ABD calcium dynamics during 371 slow phase. dcaml1 mutant animal's performance dropped off at higher speeds, but the effects were 372 relatively mild. Given the essential role of the retinal $\mathrm{ON}$ pathway in $\mathrm{OKR}^{48}$ and the diminished 373 behavioral responses to light onset (locomotor and background adaptation), the normal slow phase 374 performance may be due to compensatory mechanisms that overcome reduced retinal sensory 375 input. It is also worth noting that torsional VOR was affected more strongly than horizontal OKR, 376 indicating that dscaml1's effect on different subcircuits of the oculomotor system is not uniform.

377 Loss of dscaml1 resulted in a notable deficit in the function of the integrator pathway. This is most 378 clearly seen in the fast decay of eye fixation after spontaneous saccade in dscaml1 mutants, relative 379 to controls. Additionally, slow phase deficits in mutants may partly arise from the integrator 380 pathway, which is necessary to integrate the velocity signal from the vestibular nuclei to encode a 381 smooth ramp of eye position ${ }^{50}$. The behavioral dysfunction observed in the mutant could arise from 382 a deficit in the circuit connectivity within the integrator for supporting and coordinating persistent 383 firing $45,51$.

384 Lastly, the pronounced time-dependent fatigue and lock up phenotypes in dscaml1 mutants are 385 likely contributed by a combination of different pathways and broader effects beyond the 386 oculomotor circuit. Decreased neurotransmitter release from optic nerve terminals and habituation 387 of retinorecipient neurons have previously been shown to degrade visual response under 388 prolonged stimulation; these mechanisms may underlie the reduced robustness in dscaml1 mutants 389 35, 36. Similarly, lock up may result from changes in neuronal excitability and seizure-like episodes, 390 analogous to what was seen in the zebrafish didy mutants (see next section) ${ }^{34}$.

391 Comparison with didy (Nav1.1b) mutants

392 Some aspects of the dscaml1 phenotype are similar to a previously described zebrafish mutant, didy, 393 which encodes the voltage-gated sodium channel Scn1lab (Nav1.1b) 34. didy mutants have 
spontaneous seizure-like brain activity and very infrequent spontaneous saccades. After 15 seconds of continuous OKR stimulation, didy mutants cease to initiate resetting saccades, resulting in lock up of the eyes. Another similarity between didy and dscaml1 is defective light adaptation (darker pigmentation, slow response to light stimulus). didy and dscaml1 may both affect the saccadic pathway, which progressively loses excitability in didy mutants. It is important to note, however, that the saccade phenotypes are distinct between didy and dscaml1. In didy animals, saccades have normal speed and amplitude ${ }^{34}$, whereas saccades in dscaml1 animals are slow and small. 401 Furthermore, lock-up events in dscaml1 mutants occur more sporadically and across the position 402 range, whereas lock up only occurs at the most eccentric position at the end of a slow phase in didy mutants. These distinctions suggest that didy and dscaml1 likely affect saccade generation through independent mechanisms

Relevance to human oculomotor disorder

dscaml1 mutant fish share several features of COMA: failure to initiate saccadic eye movements, hypometric saccades, normal main sequence, and intermittent lock up during horizontal OKR. COMA is an infantile-onset condition involving failure of both voluntary and reflexive saccadic eye movements $16,17,52$. As it is not a true apraxia (where only voluntary movements are affected), the condition is also known as intermittent saccade initiation failure or infantile-onset saccade initiation delay. The etiology of COMA is still poorly understood. Genomic regions surrounding the causative gene for juvenile nephronophthisis (NPHP1) have been suggested to contribute to COMA, but mutations in NPHP1 itself do not consistently cause COMA 53 . NPHP1 has been linked to primary cilia function, which is crucial for the development of the cerebellum, a key region involved in saccade control $54,55,56$. Although there is currently no genetic association between human DSCAML1 416 and COMA (DSCAML1 resides on a separate chromosome from NPHP1), further analysis of the 417 dscaml1 mutants (e.g., cerebellum and extraocular motor neurons) may provide insights to the 418 pathogenesis of COMA and the development of neural circuitry for saccades in general.

\section{Conclusions}

420 Our investigations on zebrafish dscaml1 revealed essential roles for a DSCAM family gene in 421 visuomotor behavior and subcircuit activity. Given the structural conservation of subcortical 
422 circuits and the functional conservation of dscaml1's roles in retinal patterning, it is plausible that

423 the mammalian DSCAML1 will also contribute to visuomotor processing. By taking advantage of the

424 translucent larval zebrafish system, we recorded oculomotor circuit output dynamics in behaving 425 animals and uncovered a specific dependence of the saccade pathway on $d s c a m l 1$. Our physiological 426 findings in both control and mutant contexts provide a neural basis for the saccade deficits seen in 427 dscaml1 mutants and potentially for human saccade palsy (COMA). In addition to saccade deficits, 428 our broad examination of oculomotor behaviors also revealed dscaml1's function in VOR, neural 429 integration, and behavioral robustness. These behavioral characterizations provide links between 430 dscaml1 and diverse aspects of sensorimotor function and will facilitate future studies on the 431 development and disorders of sensorimotor circuits.

\section{Materials and Methods}

Zebrafish husbandry

435 Zebrafish (all ages) were raised under $14 \mathrm{~h}$ light/10h dark cycle at $28.5^{\circ} \mathrm{C}$. Embryos and larvae were 436 raised in water containing 0.1\% Methylene Blue hydrate (Sigma-Aldrich). At 24 hours post437 fertilization, embryos used for histological analyses were transferred to E3 buffer containing 438 0.003\% 1-phenyl-2-thiourea (PTU; Sigma-Aldrich) to prevent pigment formation. Developmental 439 stages are as described by Kimmel et al. ${ }^{57}$. All experimental procedures are performed in 440 accordance with Institutional Animal Care and Use Committee guidelines at Augusta University, 441 Virginia Tech, and Weill Cornell Medical College.

\section{$442 \quad$ Mutant and Transgenic Zebrafish lines}

443 dscaml1 (ZFIN gene name: Down syndrome cell adhesion molecule like 1) mutant was generated in 444 TL/AB mixed background using TAL effector nucleases (TALENs) as previously described 21,58. Two 445 alleles were identified, one harbored a 6 base pair insertion (in frame) and the other harbored a 7 446 base pair deletion (frame shift). The 7 base deletion mutant (dscaml1vt1) was used for further 447 analysis. The dscaml1 ${ }^{\text {vt1 }}$ allele generates a HaeIII (New England Biolabs) restriction site, which was 448 used to distinguish between wild-type and dscaml1 ${ }^{\text {tt } 1}$ alleles. DNA prep and PCR were performed as 
449 described previously59, followed by HaelII digestion for 2 hours at $37^{\circ} \mathrm{C}$ (primer sequences:

450 aaatactgcacggtgcacacgtc and atgcagatcctacagcctcataatc). After HaeIII digestion, wild-type band was 451395 base pairs (uncut) whereas mutant bands were 315 and 75 base pairs. Sequencing of $d s c a m l 1$ 452 transcript confirmed incorporation of the 7-base deletion into the open reading frame. 453 Tg(atoh7:GAP-RFP) animals were provided by Owen Randlett (Harvard University, Cambridge, MA, 454 USA)28. Tg(elavl3:H2B-GCaMP6f) and casper (nac-/-;roy-/-) animals were provided by Misha Ahrens 455 (HHMI Janelia Farm Research Campus, Ashburn, VA, USA) ${ }^{46}$.

Image acquisition and processing

457 Imaging procedures were as previously described ${ }^{60}$. Fluorescent images were acquired using an 458 Olympus FV1000 laser-scanning confocal system with a 20x XLUMPlanFl water-immersion 459 objective or a Nikon A1R MP+ laser scanning confocal system with a CFI75 Apochromat LWD 25x 460 water-immersion objective. Larvae were immobilized with 0.01\% tricaine methanesulfonate (MS461 222, Sigma-Aldrich), embedded in molten 1.5\% low-melt agarose (Fisher Scientific) in a glass462 bottomed Petri dish (P50G-1.5-14-F, MatTek). Fish were mounted so that the surface to be imaged 463 was facing the glass bottom.

464 Images were processed with Fiji ${ }^{61}$ and Photoshop (Adobe Systems) software. To measure the extent 465 of serotonergic amacrine cell aggregation, we acquired confocal stacks and determined the center 466 of mass for each 5-HT positive cell in ( $\mathrm{x}, \mathrm{y}, \mathrm{z})$ coordinates. Intercellular distance between two cells 467 was calculated by the distance formula: $d=\sqrt{\left(x_{2}-x_{1}\right)^{2}+\left(y_{2}-y_{1}\right)^{2}+\left(z_{2}-z_{1}\right)^{2}}$. For each cell, 468 intercellular distance to all other cells in the same retina were calculated. Cells with neighboring 469 cells within 3 cell diameter ( $\leq 10$ microns) were categorized as aggregated. For each eye, the number 470 of aggregating cells was divided by the total number of cells to calculate aggregation ratio.

471 Quantification of the distribution of PKC $\alpha$ immunolabeling was performed as described by Nevin et $472 a{ }^{27}$ Using Fiji, a rectangle was drawn across a relatively flat section of the IPL so that the top and 473 bottom of the rectangle abut the boundaries of the IPL, as defined by SV2 immunolabeling. Next, the 474 Plot Profile function was used to measure the average fluorescence intensity across the thickness 475 of the IPL. After exporting numerical data to Excel (Microsoft Inc.), data were normalized to maximal 
476 fluorescence intensity and relative position within the IPL. The number of serotonergic neurons 477 were estimated by manually counting $5 \mathrm{HT}+$ cells from 7 optical sections $(10 \mu \mathrm{m}$ apart, at horizontal 478 levels adjacent to the lens) in each fish.

479 For 3D rendering of retinal afferents, $\mathrm{Tg}$ (atoh7:GAP-RFP) transgenic fish were fixed with 4\% PFA 480 and mounted laterally. The eye on the imaged side was removed to allow visualization of the optic 481 tract. 3D rendering was created using Nikon NIS-Elements software. Measurements of images were 482 analyzed using the Prism 6 statistic software (GraphPad).

484 dscaml1 probe was generated by 5'RACE (Smart RACE cDNA Amplification Kit, Clonetech) using 3' 485 primers designed from Ensembl exon predictions. Amplified DNA was cloned into the pCRII-TOPO 486 vector by TA cloning (Invitrogen). Probe sequence includes 127 base pairs of 5' untranslated region 487 and 545 base pairs of coding sequence. DIG-labeled dscaml1 probe synthesis and whole mount in 488 situ hybridization were performed as previously described ${ }^{60}$. For 3 and 5 days post-fertilization 489 (dpf) samples, Dextran sulfate (Sigma-Aldrich) and 4-iodophenol (Fluka) were added to the 490 hybridization and tyramide solution to increase signal intensity 62. Whole-mount 491 immunohistochemistry was performed as described by Randlett et al. 63. Primary antibodies used 492 were: Znp-1 (anti-synaptotagmin2, Developmental Studies Hybridoma Bank), anti-acetylated493 tubulin (Sigma), anti-SV2 (Developmental Studies Hybridoma Bank), anti-HuC/D (Invitrogen), anti494 HNK-1 (zn-12, Developmental Studies Hybridoma Bank), anti-5-HT (Sigma), anti-PKC $\alpha$ (Santa Cruz 495 Biotechnologies), zpr-1 (Zebrafish International Resource Center), and anti-Blbp (Abcam). Alexa 496 fluor-conjugated secondary antibodies were used after primary antibody incubation.

\section{Locomotor assay}

498 Individual 5 dpf larvae were placed into each well of a 24-well tissue culture plate (Fisher Scientific) 499 and transferred into a Zebrabox imaging chamber (Viewpoint). Locomotor activity of each larva was 500 tracked over 24 hours, with white LED illumination turned off at $10 \mathrm{pm}$ and on at 8 am. Total 501 displacement over time was integrated every 10 minutes, measured as previously described 31. 


\section{$\underline{\text { OKR and saccade assays }}$}

503 VisioTracker 302060 (New Behavior TSE) was used for OKR and saccade assays. Eye movements of 504 individual fish were recorded by an overhead CCD camera. Zebrafish larvae were placed in the 505 center of a 50mm glass bottom petri dish (MatTek) and immobilized in 1.5-2\% low melting agarose 506 (Fisher Scientific) in E3 buffer. Agarose around the eye was removed to allow free eye movement. 507 The dish was then filled with E3. To test slow phase performance under short periodicity, the 508 direction of black and white grating switched every 3 seconds with grating velocity at $7.5^{\circ} / \mathrm{s}$. Each 509 experimental run (trial) was 108 seconds long and included twelve 9-second phases at varying 510 contrast levels $(0.99,1.0,0.5,0.2,0.1,0.05,0.02,0.05,0.1,0.2,0.5,1.0)$. For each animal, 5-6 trials 511 were tested and OKR response typically initiated during the first three trials (initiation trials). 512 Contrast sensitivity was calculated using trials recorded after the initiation trials. To exclude the 513 effect of response latency and the initial ramping up of eye velocity at stimulus onset, the first 1 514 second of each 3-second half period was excluded from analysis ${ }^{33}$. Spontaneous saccades and OKR 515 performance under long periodicity were tested using a trial that contains four phases: (1) uniform 516 illumination (1-160s), (2) square wave grating with direction switching every 40s, contrast=1, 517 spatial frequency $=0.05$ cycles $~^{\circ}$, and velocity $=10^{\circ} / \mathrm{s}(161-400 \mathrm{~s})$, (3) uniform illumination (401518 480s), (4) square wave grating with direction switching every 8s, contrast=1, spatial 519 frequency $=0.05$ cycles ${ }^{\circ}$, and velocity $=10^{\circ} / \mathrm{s}(481-560 \mathrm{~s})$.

520 A saccadic event was defined as sample periods where the instantaneous absolute eye velocity is 521 greater than $50^{\circ}$ s. Slow-phase eye velocity was measured as the mean, saccade-removed, 522 instantaneous velocity averaged across time within 1-second bins. Instantaneous velocity was 523 measured as the difference in smoothed eye position divided by the sample period (200ms, $5 \mathrm{~Hz})$. 524 Eye position was smoothed using a median filter (Matlab medfilt1) of 5 sample periods (1s). We 525 excluded sample periods that fell between 0.5 seconds before and 2 seconds after each saccade to 526 remove the effects of saccade filtering and post-saccade plant relaxation ${ }^{64}$. To analyze lock-up 527 probability and saccade conjugacy we measured eye velocity independently for each eye since lock528 up could occur independently on a given eye. Lock up periods are defined as 1-second bins where 529 average velocity is $<1^{\circ} /$ s. Lock up probability was defined as the average number of lock up periods 530 across trials and both eyes. We defined a disconjugacy index as the ratio of left and right 
531 instantaneous eye velocity magnitude at each time bin where a saccadic event occurs. The index is

532 defined so that the larger saccade velocity is in the numerator which means the index is always 533 greater than or equal to 1 . For all other plots, instantaneous velocity was averaged across both eyes 534 before binning.

535 To calculate the main sequence of saccade, eye positions were recorded at $30 \mathrm{~Hz}$ (33 ms sample 536 period). Saccade peak velocity and amplitude were measured as described by Chen et al. ${ }^{41}$ The main 537 sequence was calculated as the slope of the linear regression for peak velocity and amplitude, using 538 the Prism6 software (Graphpad) ${ }^{19}$.

539 To measure the drift rate in eye position following spontaneous saccades during the uniform 540 illumination period of the stimulus, we used a quasi-Newton unconstrained optimization (Matlab 541 fminunc with Algorithm set to quasi-Newton) to minimize the squared error between eye position 542 and the function

$543 A e^{-t / \tau}+E_{0}$

544 with variable parameters $\mathrm{A}$ and $\tau$. $E_{0}$ was fixed to the mean position for the eye being fit. The 545 algorithm was initialized with tau set to 10 seconds and A set to the first eye position value in the 546 sample. We excluded positions that occurred between 0-1 seconds after each saccade, to avoid 547 fitting post-saccadic relaxation related to plant mechanics. Since the duration of spontaneous 548 fixations was variable, the fixation window analyzed was variable with typical values between 5-20 549 seconds. We only analyzed exponential fits that passed the following goodness-of-fit criteria: sum550 of-squared errors was less than $\left(30^{\circ}\right)^{2}$ or one minus the ratio of mean squared-error to the sample 551 variance was greater than 0.4 and the sum-of-squared errors was less than $\left(200^{\circ}\right)^{2}$. These criteria 552 were chosen based on visual inspection of fit qualities.

\section{$553 \quad$ VOR assay}

554 Torsional eye movements were measured in 5 days post-fertilization fish in response to step tilts 555 delivered using an apparatus similar in design to Schoppik et al. 2017 38. All experiments took place 556 in the dark. Larval fish were immobilized completely in 2\% low-melting-temperature agar (Thermo 
557 Fisher), and the left eye freed. The agar was then pinned (0.1mm stainless minutien pins, FST) to a $558 \sim 5 \mathrm{~mm}^{2}$ piece of Sylgard 184 (Dow Corning) which was itself pinned to Sylgard 184 at the bottom 559 of a $10 \mathrm{~mm}^{2}$ optical glass cuvette (Azzota). The cuvette was filled with $1 \mathrm{ml}$ of E3 media and placed 560 in a custom holder on a 5-axis (X, Y, Z, pitch, roll) manipulator (ThorLabs MT3 and GN2). The fish 561 was aligned with the optical axes of two orthogonally placed cameras such that both the left utricle 562 and two eyes were level with the horizon (front camera). The experimenter running behavior was 563 blind as to the genotype of the fish.

564 The eye-monitoring camera (Guppy Pro 2 F-031, Allied Vision Technologies) used a 5x objective 565 (Olympus MPLN, $0.1 \mathrm{NA}$ ) and custom image-forming optics to create a 100x100 pixel image of the 566 left eye of the fish $(6 \mu \mathrm{m} /$ pixel), acquired at $200 \mathrm{~Hz}$. The image was processed on-line by custom 567 pattern matching software to derive an estimate of torsional angle (LabView, National Instruments), 568 and data were analyzed using custom MATLAB scripts. A stepper motor (Oriental Motors AR98MA569 N5-3) was used to rotate the platform holding the cameras and fish. The platform velocity and 570 acceleration were measured using integrated circuits (IDG500, Invensense and ADXL335, Analog 571 Devices) mounted together on a breakout board (Sparkfun SEN-09268). Fish were rotated stepwise 572 for 4 cycles: from $0^{\circ}$ to $-15^{\circ}$, where positive values are nose-down, then from $-15^{\circ}$ to $0^{\circ}$, from $0^{\circ}$ to $57315^{\circ}$, then back to $0^{\circ}$. Steps had a peak velocity at $35^{\circ} / \mathrm{sec}$. The inter-step interval was 7.5 seconds.

574 The eye's response across the experiment was first centered to remove any offset introduced by the 575 pattern-matching algorithm. Data were then interpolated with a cubic spline interpolation to 576 correct for occasional transient slowdowns (i.e., missed frames) introduced by the pattern577 matching algorithm. The eye's velocity was estimated by differentiating the position trace; high578 frequency noise was minimized using a 4-pole low-pass Butterworth filter (cutoff $=3 \mathrm{~Hz}$ ). Each step 579 response was evaluated manually; trials with rapid deviations in eye position indicative of 580 horizontal saccades or gross failure of the pattern-matching algorithm were excluded from analysis. 581 Across all fish and all steps used to measure the behavior, the median number of usable responses 582 was 7/10. The response to each step for a given fish was defined as the mean across all responses 583 to that step across cycles. The gain was estimated by measuring the peak eye velocity occurring over 584 the period 375-1000ms after the start of the step. Only steps away from the horizon were analyzed. 
585 Of 9 fish, one was excluded because it had fewer than ten steps for analysis, all others had at least 586 ten. The median number of steps \pm interquartile range was 18/15 $\pm 13.75 / 10.5$ for nose-down/nose587 up steps respectively.

Two-Photon Calcium Imaging during Behavior

589 Embryos from crosses of dscaml1 heterozygous mutants, (dscaml1+/-;casper+/- X dscaml1+/590 ;nac+/-; elavl3:H2B-GCaMP6f) were used. At 5-7 dpf, pigmentless (nac-/-) dscaml1 heterozygous and 591 homozygous mutant siblings were immobilized in a gel of 1.8\% low-melting temperature agarose 592 (Sigma-Aldrich) in preparation for imaging. Agarose was removed from the eyes to allow them to 593 move freely during imaging. Each fish was genotyped following imaging. Simultaneous eye tracking 594 and two-photon calcium imaging were performed using a custom-built system as previously 595 described ${ }^{13}$. Each image was acquired by raster scanning a mode-locked excitation laser 596 (wavelength set to 930 nanometers) through a 40x water immersion lens to a horizontal plane at 597 the abducens motor neurons. The laser power at the sample varied between 15-25 mW. For each 598 animal, we recorded 3-10 planes at a rate of $1.95 \mathrm{~Hz}$ and duration of 5 minutes per plane. Each plane 599 was recorded while vertical stripes were projected onto a screen of diffusion film placed 1-3 cm in 600 front of the animal providing an optokinetic stimulus ${ }^{13}$. The stripes moved at a constant velocity 601 whose magnitude and direction changed in a repeating pattern that consisted of equal durations of 602 positive, negative, and zero velocity, with each phase lasting 3.14 (2 heterozygotes and 6 mutants) 603 or 31.26 seconds ( 1 heterozygotes and 1 mutant). Eye position was measured using a sub-stage, 604 infrared camera (Allied Vision Technologies, Guppy FireWire camera) that acquired frames at 13 $605 \mathrm{~Hz}^{13}$.

\section{Identification of putative abducens neurons}

607 Putative abducens neurons were identified based on cell location and fluorescence activity. Abducens motor neurons are clustered in rhombomeres (rh) 5-6 and are arranged in dorsal-ventral columns. The imaging window $\left(185 \mu \mathrm{m}^{2}\right)$ was positioned over rh 5-6 using the posterior otolith as guides to image the abducens population ${ }^{65}$. We only examined cells whose fluorescence responses were correlated with direction-rectified eye-position and/or eye-velocity traces with an absolute 
612 Pearson correlation coefficient greater than or equal to $0.313,44,51$. Before correlation calculation, 613 we convolved eye-position and eye-velocity variables with a 2 second exponentially decaying 614 calcium impulse response function to account for the calcium buffering associated with a cell's 615 action potential 44,66. Single neurons that contained at least one pixel with an absolute correlation 616 value above 0.3 were manually selected for further analysis. To correct for animal motion artifacts, 617 fluorescence movies were first pre-processed using a procedure that relies on cross-correlation of 618 individual frames with a time-averaged reference frame ${ }^{51}$. Frames that undergo large shifts from 619 the reference frame (greater than the median plus 5 times the median absolute deviation) were 620 excluded from analysis. To compute delta $F$ over $F$ time series (dF/F), we subtracted and then 621 divided the time-averaged fluorescence within each ROI.

623 For each cell, we computed the average $\mathrm{dF} / \mathrm{F}$ response relative to saccadic (fast-phase) eye 624 movements and relative to the start of optokinetic stimulus movements. A fast-phase event is 625 defined as described above (50\%). Eye velocity is computed as the instantaneous difference in 626 smoothed eye position divided by instantaneous eye sampling time (77 ms). Eye position was 627 smoothed using a median filter (Matlab medfilt1) of order equivalent to $500 \mathrm{~ms}$. In order to combine $628 \mathrm{dF} / \mathrm{F}$ traces across saccadic events and the start of stimuli, we linearly interpolated saccade or 629 stimulus-aligned responses to a grid of evenly spaced time bins $333 \mathrm{~ms}$ in width before averaging. 630 We excluded planes from analysis if there are less than 5 saccades events available for computing 631 the STA. When averaging stimulus-aligned responses we only used traces where a fast-phase event did not occur within the first 3 seconds following stimulus onset ( $n=3$ hets, $n=6$ mutants).

\section{Acknowledgments}

635 This work was supported by funding from the National Institutes of Health (R01 EY024844 to Y.A.P., 636 K99 EY027017 to A.D.R., R01 EY027036 to E.R.F.A.), the Medical College of Georgia, and Virginia 637 Tech. We thank the animal care staff at Augusta University and Virginia Tech for animal husbandry, 638 the Augusta University Electron Microscopy and Histology Core for retina histology preparation, A. 
639 Pauli for help generating the dscaml1 TALEN mutants, J. Mathias for assistance with locomotor 640 behavior analysis, F. Ali for technical assistance, and K. Bollinger and J. Sanes for helpful discussions.

\section{Author Contributions}

642 T.W., M.M., and Y.A.P. conceived the study, with input from A.F.S. Y.A.P., J.A.G. and S.Z. generated the 643 dscaml1 mutant line, using TALEN targeting constructs designed and made by D.R., S.Q.T., and J.K.J.

644 T.W., M.M., R.R., C.K., and Y.A.P. contributed to the histological analyses. M.M., A.R. A.S., K.E.H., D.S., 645 E.R.F.A. and Y.A.P. contributed to the behavioral analyses. A.R. and E.R.F.A. designed and interpreted 646 the functional imaging experiments. A.R. performed and analyzed the functional imaging 647 experiments with help from M.M. Y.A.P. wrote the manuscript, with contributions from T.W., M.M., 648 A.R., R.R., D.S., S.L.G., and E.R.F.A.

Movie 1. OKR response. Control (left) and dscaml1-/- (right) animals performing OKR.

651 Figure 1. The Oculomotor circuit, and the expression and gene targeting of dscaml1. A, 652 Diagram of the oculomotor circuit for horizontal eye movement. Visual motion activates directional 653 selective retinal ganglion cells, which innervate the optic tectum (OT) and pretectum (PT). PT 654 provides input to the vestibular nucleus (VN). OT (along with other areas), activates the excitatory 655 burst neurons (EBN). EBN and VN provide premotor input to the abducens nucleus (ABD), which 656 innervates the extraocular muscles. EBN and VN also innervate the velocity-position neural 657 integrator (NI), which provides an eye position signal to the ABD. The saccadic, vestibular, and 658 integrator pathways are labeled light brown, dark brown, and blue, respectively. B-G, dscaml1 $659 m R N A$ (white) labeled by fluorescent in situ hybridization, with neuropil counterstained with an 660 antibody against synaptotagmin 2 (Znp-1, magenta). Developmental staging as indicated. $\mathbf{H}$, 661 Alignment of wild-type and TALEN targeted dscaml1 genomic sequence. The start codon is boxed, 662 and the region containing insertions and deletions is highlighted in red. I, Pigmentation pattern of 663 different genotypes. dscaml1 mutant animals show darker overall pigmentation. J, Survival curve, 664 sorted by genotype. GCL: ganglion cell layer; INL: inner nuclear layer; ONL: outer nuclear layer. Scale 665 bar is $100 \mu \mathrm{m}$ in panel $\mathrm{A}-\mathrm{F}, 1 \mathrm{~mm}$ in $\mathrm{H}$. 
666 Figure 2. dscaml1 is required for planar and laminar patterning of the retina. A, H\&E staining 667 of 5 dpf retina. B, Quantification of IPL thickness, performed in confocal imaged 5 dpf larvae 668 immunostained with Znp-1 antibody. Mutants have significantly thicker IPL compared to wild-type 669 animals. Heterozygotes have an intermediate phenotype. C, Serotonergic amacrine cells, stained 670 with an antibody against 5-hydroxytryptamine (5-HT). Cell bodies are indicated by yellow 671 arrowheads. D, Percentage of cells that are immediately adjacent to another cell (within $10 \mu \mathrm{m}$, 672 approximately 3 cell diameter) is significantly higher in $d s c a m l 1-/-$ animals (**p<0.01, one-way 673 ANOVA). E, The number of 5-HT positive amacrine cells was not significantly different. F, 674 Immunostaining of ON-bipolar cell ( $\mathrm{PKC} \alpha$, green), retinal ganglion cell [Tg(atoh7:GAP-RFP), red], 675 and synapses (SV2, blue). Boxed areas are enlarged two-fold and shown in the inset. Both mutants 676 and morphants show loss of discrete stratification and mature terminal boutons in the ON (lower) 677 sublamina of the IPL. G, PKC $\alpha$ immunolabeling intensity (vertical axis) was plotted across the depth 678 of the IPL (horizontal axis), normalized for maximal intensity and IPL thickness. Three prominent 679 peaks can be discerned in wild-type and heterozygote animals (red arrowheads). The number and 680 location of peaks are more variable in mutants and morphants. GCL: ganglion cell layer; INL: inner 681 nuclear layer; IPL: inner plexiform layer; ONL: outer nuclear layer; OPL: outer plexiform layer. Scale 682 bars are $25 \mu \mathrm{m}$. One-way ANOVA was used for statistical comparisons in D and E (****p<0.0001, $683 * * * \mathrm{p}<0.001, * * \mathrm{p}<0.01)$.

684 Figure 3. Normal development of Müller glia, outer retina, and optic tract in the dscaml1 685 mutant. A-F, No abnormalities were seen in cone photoreceptor cells (stained with zpr-1 antibody) 686 and Müller glia (anti-Blbp). G-I, Retinal afferent projection in dscaml1 mutants. The panel above G 687 shows an illustration of retinal afferents (red) in the $\mathrm{Tg}($ atoh7:GAP-RFP) transgenic. Images show 688 the 3D reconstruction of retinal afferents viewed from the side (left images) and front (right 689 images). Retinal arborization fields (AFs) in the thalamus (AF4), pretectum (AF7, 9), and optic 690 tectum (AF10) are identified as described previously ${ }^{29}$. No differences were observed among 691 different genotypes. OT: optic tract. Panels A-F are shown at the same scale, scale bar in A is $25 \mu \mathrm{m}$. 692 Panels G-I are shown at the same scale, scale bar in G is $100 \mu \mathrm{m}$. 
693 Figure 4. Locomotor activity in response to light. A, Locomotor activity over 24 hours. Solid lines 694 are mean movement ( $\mathrm{n}=8$ for each group), and dotted lines indicate the range of standard error. 695 Lighting conditions are indicated on the X-axis, with each tick marking one hour. B-C, Percentage 696 time active during the day (B) and night (C). D-E, Average movement during the day (D) and night

697 (E). F-G, Total amount of movement 30 minutes after lights switch on (F) and off (G). *p<0.05 for 698 pairwise comparisons using one-way ANOVA.

699 Figure 5. OKR and VOR performance in dscaml1 mutants. A, larvae were immobilized in the 700 center of a circular arena, where black and white vertical bars are projected. Diagram of eye position 701 is shown on the right, with fast phases (red segments) and slow phases (blue segments). B-E, short 702 time-scale OKR. Eye position traces are shown for control (C) and dscaml1-/- (D) animals, at the 703 same time scale as the square wave stimulus (B). Right and left eye traces are in black and fuchsia, 704 respectively. E-G, long time-scale OKR. Eye velocity and position traces are shown for control (C) 705 and $d$ scaml1-/- (D), at the same time scale as the stimulus (E). In mutants, the eyes intermittently 706 became locked up (red arrowheads). H, slow-phase velocity across a range of contrast levels. $707\left({ }^{*} \mathrm{p}<0.05\right.$, two-way ANOVA with Bonferroni correction). I, saccade amplitude is lower in mutants $708\left(^{* * * *} \mathrm{p}<0.0001\right.$, two-sample t-test). J-K, the temporal dependency of lock-up probability (J), slow709 phase gain $(\mathrm{K})$, and slow-phase gain excluding lock-up periods (L). Positive gains in $\mathrm{H}, \mathrm{J}-\mathrm{K}$ are 710 defined as eye movement in the same direction as the stimulus. Regression lines, means, and 711 standard error (shaded areas) are shown. Linear regression model was used in J and control group 712 in K. Two phase exponential decay regression model with plateau constrained to zero was used for 713 dscaml1-/- group in Kand both groups in L. M, eye velocity in response to a $35^{\circ} / \mathrm{s}$ tilt stimulus (black 714 dotted line) in wild-type (top panel, solid black line) and dscaml1 mutant (bottom panel, solid red 715 line). Shaded areas indicate interquartile range. Torsional velocity was slower in mutants, 716 compared to wild type. N, velocity gain of upward and downward tilts, both of which are lower in 717 mutants. Mean values are marked by open circles. ${ }^{*} \mathrm{p}<0.05$, Mann-Whitney $U$ test.

718 Figure 6. Spontaneous saccade and fixation are abnormal in dscaml1 mutants. A-B, 719 spontaneous saccades in control (A) and dscaml1-/- (B) were recorded during uniform illumination 720 (no gratings). Right and left eye traces are in black and fuchsia, respectively. Red arrowheads 721 indicate disconjugated saccades. C, the frequency of eye movements divided into angular velocity 
722 bins. Controls had significantly more eye movements greater than $100^{\circ} / \mathrm{s}$ compared to mutants,

723 whereas mutants had more eye movements in the slowest bin compared to control $\left({ }^{*} \mathrm{p}<0.05\right.$, $724^{* * * *} \mathrm{p}<0.0001$, two-way ANOVA). D, the relative frequency distribution of eye movements based on 725 angular velocity. Control animals perform significantly more fast eye movements than mutants 726 ( $\mathrm{p}<0.0001$, two-sample K-S test). $\mathbf{E}$, cumulative distribution of saccade disconjugacy index for 727 controls and mutants (higher equals more disconjugated, see methods). Control index has a median 728 value of 1.56 across the population while the mutant index has a higher median value of 2.33 . 729 Distribution is significantly different between control and mutant ( $\mathrm{p}<0.001$, two-sample K-S test). 730 F, peak velocity and amplitude for each saccade event was plotted, along with linear regression 731 (solid line). The slope is not significantly different between control and mutant ( $\mathrm{p}=0.46)$. G-H, an 732 example of TagRFP-T expressing abducens motor neurons [from $\operatorname{Tg}(m n x 1$ :TagRFP-T)] in wild-type 733 (G) and mutant (H) animal. Dorsal view, rostral to the left. Labeled structures: motor axons (VIth 734 nerve, white arrows), rostral abducens complex (green arrowheads), and caudal abducens complex 735 (magenta arrowheads). Scale bar is $100 \mu \mathrm{m}$. I, eye position decay (arrows in A, B) calculated as $1 / \tau$. ****: p<0.0001, Mann-Whitney $U$ test.

Figure 7. Two-photon calcium imaging in Abducens and Inferior Olive neural populations. AD, two-photon calcium imaging and simultaneous eye position recording during OKR. Circles in time-averaged images show the locations of cells (A, B) with corresponding indices whose fluorescence activity is shown (C, D). Circles in the abducens motor complex (ABD) are marked. E, average fluorescence traces of individual cells (Y-axis) aligned to stimulus onset during slow-phase activity (X-axis) in the ABD. F, Population average of fluorescence traces in E. G, average fluorescence traces aligned to fast-phase eye movements for cells in the ABD. $\mathbf{H}$, activity in $G$ averaged across the population. Error bars in $\mathrm{F}$ and $\mathrm{H}$ show the median absolute deviation of singlecell activities from the population average divided by the square root of the number of cells, normalized so that population responses are between -1 and 1 .

747 Supplementary Figure S1. Brain morphogenesis is grossly normal in dscaml1 mutants. A-B, 748 Lateral view of axon tracts (Ac-tub, green) and synapses (znp-1, magenta) at 1 and $5 \mathrm{dpf}$. In $1 \mathrm{dpf}$ 749 embryos (A), no differences were seen in the formation of the major commissures and longitudinal 
750 tracts ${ }^{67}$. In $5 \mathrm{dpf}$ larvae, no apparent abnormalities were seen in the morphology of sensory nerves,

751 motor nerves, or distribution of synapses in the brain and retina 68, 69. C, Lateral view of the 752 neuromuscular junction of the trunk, stained with a marker for presynaptic terminals (SV2). D. 753 Dorsal view of $5 \mathrm{dpf}$ larvae stained for mature neurons (Hu, magenta) and axon tracts (HNK-1, 754 green). Areas outlined in yellow are the optic tectum neuropil region. Images show maximal 755 intensity projection of confocal image stacks. Scale bars are $100 \mu \mathrm{m}$.

756 Supplementary Figure S2. Eye Position during lock-up in dscaml1 mutants. Histogram of left 757 and right eye positions averaged during 1 second bins where lock-up occurs. Bin size is 2 degrees.

758 Tables

759 Table I. Double exponential decay fit for slow phase gain.

761 References

762 1. Iossifov I, et al. The contribution of de novo coding mutations to autism spectrum disorder. $763 \quad$ Nature 515, 216-221 (2014).

2. Karaca E, et al. Genes that Affect Brain Structure and Function Identified by Rare Variant Analyses of Mendelian Neurologic Disease. Neuron 88, 499-513 (2015).

3. Blank $\mathrm{M}$, et al. The Down syndrome critical region regulates retinogeniculate refinement. $J$ Neurosci 31, 5764-5776 (2011).

5. Maynard KR, Stein E. DSCAM contributes to dendrite arborization and spine formation in the developing cerebral cortex. J Neurosci 32, 16637-16650 (2012). 
781

7. Zhang L, Huang Y, Chen JY, Ding YQ, Song NN. DSCAM and DSCAML1 regulate the radial migration and callosal projection in developing cerebral cortex. Brain Res 1594, 61-70 (2015).

8. Bruce FM, Brown S, Smith JN, Fuerst PG, Erskine L. DSCAM promotes axon fasciculation and growth in the developing optic pathway. Proceedings of the National Academy of Sciences of the United States of America 114, 1702-1707 (2017).

9. Leigh RJ, Zee DS. The neurology of eye movements, 5th edition. edn. Oxford University Press (2015).

10. Bittencourt J, et al. Saccadic eye movement applications for psychiatric disorders. Neuropsychiatr Dis Treat 9, 1393-1409 (2013).

11. Anderson TJ, MacAskill MR. Eye movements in patients with neurodegenerative disorders. Nature Reviews Neurology 9, 74 (2013).

12. Galicia CA, Sukeena JM, Stenkamp DL, Fuerst PG. Expression patterns of dscam and sdk gene paralogs in developing zebrafish retina. Molecular vision 24, 443-458 (2018).

13. Daie K, Goldman MS, Aksay ER. Spatial patterns of persistent neural activity vary with the behavioral context of short-term memory. Neuron 85, 847-860 (2015).

14. Straka H, Beck JC, Pastor AM, Baker R. Morphology and physiology of the cerebellar vestibulolateral lobe pathways linked to oculomotor function in the goldfish. J Neurophysiol 96, 1963-1980 (2006).

15. Masseck OA, Hoffmann KP. Comparative neurobiology of the optokinetic reflex. Ann $N Y$ Acad Sci 1164, 430-439 (2009).

16. Harris CM, Shawkat F, Russell-Eggitt I, Wilson J, Taylor D. Intermittent horizontal saccade failure ('ocular motor apraxia') in children. Br J Ophthalmol 80, 151-158 (1996).

17. Zee DS, Yee RD, Singer HS. Congenital ocular motor apraxia. Brain 100, 581-599 (1977).

18. Easter SS, Jr., Nicola GN. The development of eye movements in the zebrafish (Danio rerio). Dev Psychobiol 31, 267-276 (1997). 
19. Beck JC, Gilland E, Tank DW, Baker R. Quantifying the ontogeny of optokinetic and vestibuloocular behaviors in zebrafish, medaka, and goldfish. J Neurophysiol 92, 3546-3561 (2004).

20. Fuerst PG, et al. DSCAM and DSCAML1 function in self-avoidance in multiple cell types in the developing mouse retina. Neuron 64, 484-497 (2009).

21. Reyon D, Tsai SQ, Khayter C, Foden JA, Sander JD, Joung JK. FLASH assembly of TALENs for high-throughput genome editing. Nat Biotechnol 30, 460-465 (2012).

22. Wagle M, Mathur P, Guo S. Corticotropin-releasing factor critical for zebrafish camouflage behavior is regulated by light and sensitive to ethanol. J Neurosci 31, 214-224 (2011).

23. Garrett AM, Tadenev AL, Hammond YT, Fuerst PG, Burgess RW. Replacing the PDZinteracting C-termini of DSCAM and DSCAML1 with epitope tags causes different phenotypic severity in different cell populations. eLife $\mathbf{5}$, (2016).

24. Maurer CM, Schonthaler HB, Mueller KP, Neuhauss SC. Distinct retinal deficits in a zebrafish pyruvate dehydrogenase-deficient mutant. J Neurosci 30, 11962-11972 (2010).

25. Wan L, Almers W, Chen W. Two ribeye genes in teleosts: the role of Ribeye in ribbon formation and bipolar cell development. J Neurosci 25, 941-949 (2005).

26. Schroeter EH, Wong RO, Gregg RG. In vivo development of retinal ON-bipolar cell axonal terminals visualized in nyx::MYFP transgenic zebrafish. Vis Neurosci 23, 833-843 (2006).

27. Nevin LM, Taylor MR, Baier H. Hardwiring of fine synaptic layers in the zebrafish visual pathway. Neural Dev 3, 36 (2008).

28. Zolessi FR, Poggi L, Wilkinson CJ, Chien CB, Harris WA. Polarization and orientation of retinal ganglion cells in vivo. Neural Dev 1, 2 (2006).

29. Robles E, Laurell E, Baier H. The retinal projectome reveals brain-area-specific visual representations generated by ganglion cell diversity. Curr Biol 24, 2085-2096 (2014).

30. Prober DA, Rihel J, Onah AA, Sung RJ, Schier AF. Hypocretin/orexin overexpression induces an insomnia-like phenotype in zebrafish. J Neurosci 26, 13400-13410 (2006). 
857

31. Farrell TC, Cario CL, Milanese C, Vogt A, Jeong JH, Burton EA. Evaluation of spontaneous propulsive movement as a screening tool to detect rescue of Parkinsonism phenotypes in zebrafish models. Neurobiol Dis 44, 9-18 (2011).

32. Kubo F, Hablitzel B, Dal Maschio M, Driever W, Baier H, Arrenberg AB. Functional architecture of an optic flow-responsive area that drives horizontal eye movements in zebrafish. Neuron 81, 1344-1359 (2014).

33. Rinner O, Rick JM, Neuhauss SC. Contrast sensitivity, spatial and temporal tuning of the larval zebrafish optokinetic response. Invest Ophthalmol Vis Sci 46, 137-142 (2005).

34. Schoonheim PJ, Arrenberg AB, Del Bene F, Baier H. Optogenetic localization and genetic perturbation of saccade-generating neurons in zebrafish. J Neurosci 30, 7111-7120 (2010).

35. Smear MC, et al. Vesicular glutamate transport at a central synapse limits the acuity of visual perception in zebrafish. Neuron 53, 65-77 (2007).

36. Perez-Schuster V, et al. Sustained Rhythmic Brain Activity Underlies Visual Motion Perception in Zebrafish. Cell Rep 17, 1098-1112 (2016).

37. Chen CC, et al. Velocity storage mechanism in zebrafish larvae. The Journal of Physiology 592, 203-214 (2014).

38. Schoppik D, et al. Gaze-stabilizing central vestibular neurons project asymmetrically to extraocular motoneuron pools. The Journal of Neuroscience, (2017).

39. Bianco I, Kampff A, Engert F. Prey Capture Behavior Evoked by Simple Visual Stimuli in Larval Zebrafish. Frontiers in Systems Neuroscience 5, (2011).

40. Schmitt EA, Dowling JE. Early retinal development in the zebrafish, Danio rerio: light and electron microscopic analyses. J Comp Neurol 404, 515-536 (1999).

41. Chen CC, Bockisch CJ, Straumann D, Huang MY. Saccadic and Postsaccadic Disconjugacy in Zebrafish Larvae Suggests Independent Eye Movement Control. Front Syst Neurosci 10, 80 (2016).

42. Garbutt S, Harwood MR, Harris CM. Comparison of the main sequence of reflexive saccades and the quick phases of optokinetic nystagmus. Br J Ophthalmol 85, 1477-1483 (2001). 
896

43. Sparks DL. The brainstem control of saccadic eye movements. Nature reviews Neuroscience 3, 952-964 (2002).

44. Miri A, Daie K, Burdine RD, Aksay E, Tank DW. Regression-based identification of behaviorencoding neurons during large-scale optical imaging of neural activity at cellular resolution. J Neurophysiol 105, 964-980 (2011).

45. Vishwanathan A, Daie K, Ramirez AD, Lichtman JW, Aksay ERF, Seung HS. Electron Microscopic Reconstruction of Functionally Identified Cells in a Neural Integrator. Curr Biol 27, 2137-2147 e2133 (2017).

46. Vladimirov N, et al. Light-sheet functional imaging in fictively behaving zebrafish. Nat Methods 11, 883-884 (2014).

47. Hoon M, Okawa H, Della Santina L, Wong RO. Functional architecture of the retina: development and disease. Progress in retinal and eye research 42, 44-84 (2014).

48. Emran F, Rihel J, Adolph AR, Wong KY, Kraves S, Dowling JE. OFF ganglion cells cannot drive the optokinetic reflex in zebrafish. Proceedings of the National Academy of Sciences of the United States of America 104, 19126-19131 (2007).

49. Lo CC, Wang XJ. Cortico-basal ganglia circuit mechanism for a decision threshold in reaction time tasks. Nat Neurosci 9, 956-963 (2006).

50. Cannon SC, Robinson DA. Loss of the neural integrator of the oculomotor system from brain stem lesions in monkey. J Neurophysiol 57, 1383-1409 (1987).

51. Lee MM, Arrenberg AB, Aksay ER. A structural and genotypic scaffold underlying temporal integration. J Neurosci 35, 7903-7920 (2015).

52. Salman MS. Infantile-onset saccade initiation delay (congenital ocular motor apraxia). Curr Neurol Neurosci Rep 15, 24 (2015).

53. Betz R, et al. Children with ocular motor apraxia type Cogan carry deletions in the gene (NPHP1) for juvenile nephronophthisis. J Pediatr 136, 828-831 (2000).

54. Jauregui AR, Nguyen KCQ, Hall DH, Barr MM. The $<$ em $>$ Caenorhabditis elegans $</$ em $>$ nephrocystins act as global modifiers of cilium structure. The Journal of Cell Biology 180, 973-988 (2008). 
55. Louie CM, Gleeson JG. Genetic basis of Joubert syndrome and related disorders of cerebellar development. Human molecular genetics 14 Spec No. 2, R235-242 (2005).

56. Matsui H, Namikawa K, Babaryka A, Koster RW. Functional regionalization of the teleost cerebellum analyzed in vivo. Proceedings of the National Academy of Sciences of the United States of America 111, 11846-11851 (2014).

57. Kimmel CB, Ballard WW, Kimmel SR, Ullmann B, Schilling TF. Stages of embryonic development of the zebrafish. Dev Dyn 203, 253-310 (1995).

58. Sander JD, et al. Targeted gene disruption in somatic zebrafish cells using engineered TALENs. Nat Biotechnol 29, 697-698 (2011).

59. Pan YA, et al. Zebrabow: multispectral cell labeling for cell tracing and lineage analysis in zebrafish. Development 140, 2835-2846 (2013).

60. Pan YA, Choy M, Prober DA, Schier AF. Robo2 determines subtype-specific axonal projections of trigeminal sensory neurons. Development 139, 591-600 (2012).

61. Schindelin J, et al. Fiji: an open-source platform for biological-image analysis. Nat Methods 9, 676-682 (2012).

62. Lauter G, Soll I, Hauptmann G. Multicolor fluorescent in situ hybridization to define abutting and overlapping gene expression in the embryonic zebrafish brain. Neural Dev 6, 10 (2011).

63. Randlett 0 , et al. Whole-brain activity mapping onto a zebrafish brain atlas. Nature Methods 12, 1039-1046 (2015).

64. Sklavos S, Dimitrova DM, Goldberg SJ, Porrill J, Dean P. Long time-constant behavior of the oculomotor plant in barbiturate-anesthetized primate. J Neurophysiol 95, 774-782 (2006).

65. Miri A, Daie K, Arrenberg AB, Baier H, Aksay E, Tank DW. Spatial gradients and multidimensional dynamics in a neural integrator circuit. Nat Neurosci 14, 1150-1159 (2011).

66. Kawashima T, Zwart MF, Yang CT, Mensh BD, Ahrens MB. The Serotonergic System Tracks the Outcomes of Actions to Mediate Short-Term Motor Learning. Cell 167, 933-946 e920 (2016). 
bioRxiv preprint doi: https://doi.org/10.1101/658161; this version posted June 3, 2019. The copyright holder for this preprint (which was not certified by peer review) is the author/funder, who has granted bioRxiv a license to display the preprint in perpetuity. It is made available under aCC-BY-NC-ND 4.0 International license.

975

976

977

978

979

980

981

982

983

984

985

986
67. Ross LS, Parrett T, Easter SS, Jr. Axonogenesis and morphogenesis in the embryonic zebrafish brain. J Neurosci 12, 467-482 (1992).

68. Fox MA, Sanes JR. Synaptotagmin I and II are present in distinct subsets of central synapses. J Comp Neurol 503, 280-296 (2007).

69. Higashijima S, Hotta Y, Okamoto H. Visualization of cranial motor neurons in live transgenic zebrafish expressing green fluorescent protein under the control of the islet-1 promoter/enhancer. J Neurosci 20, 206-218 (2000). 
A

A
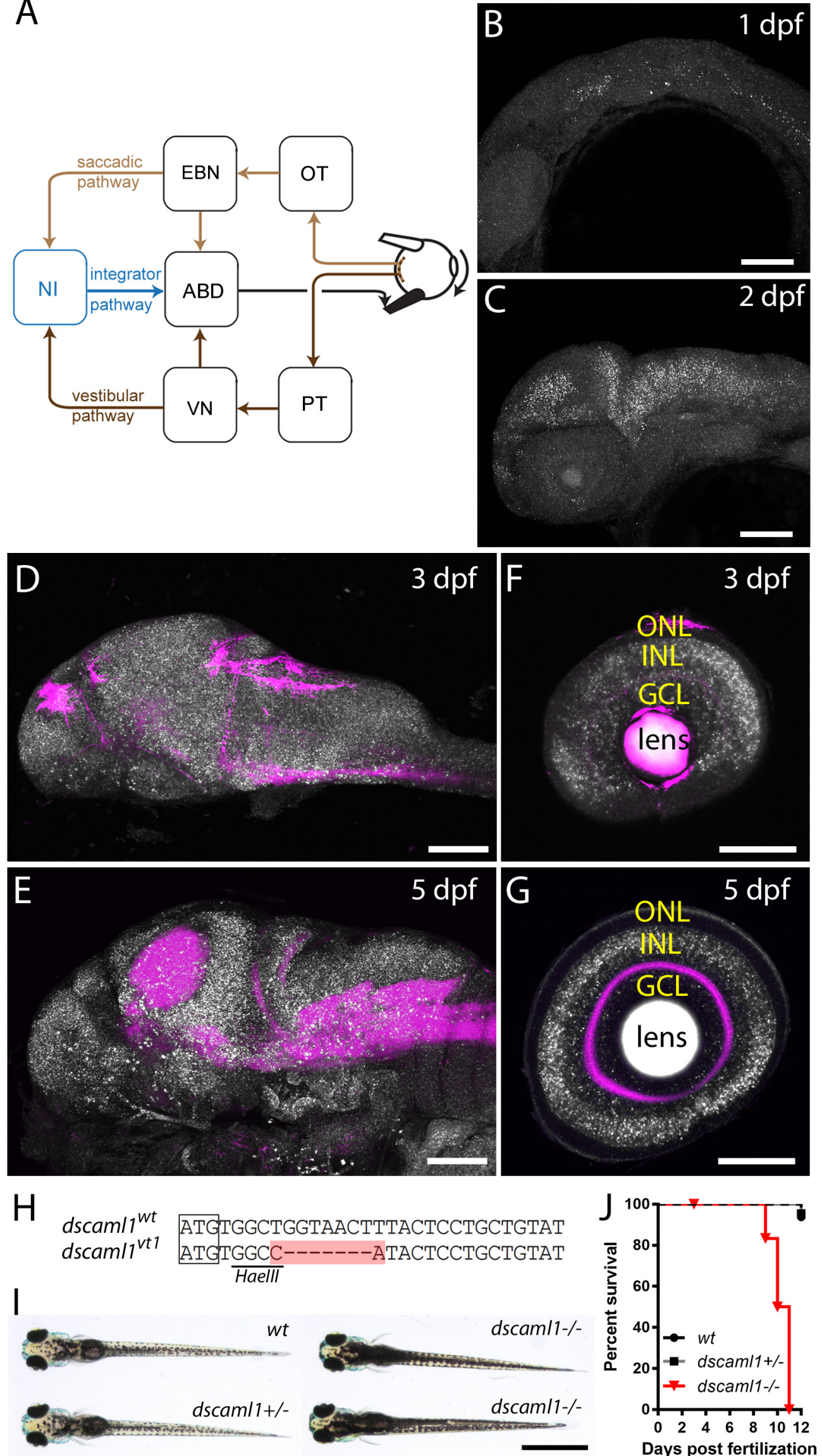

Figure 1. 


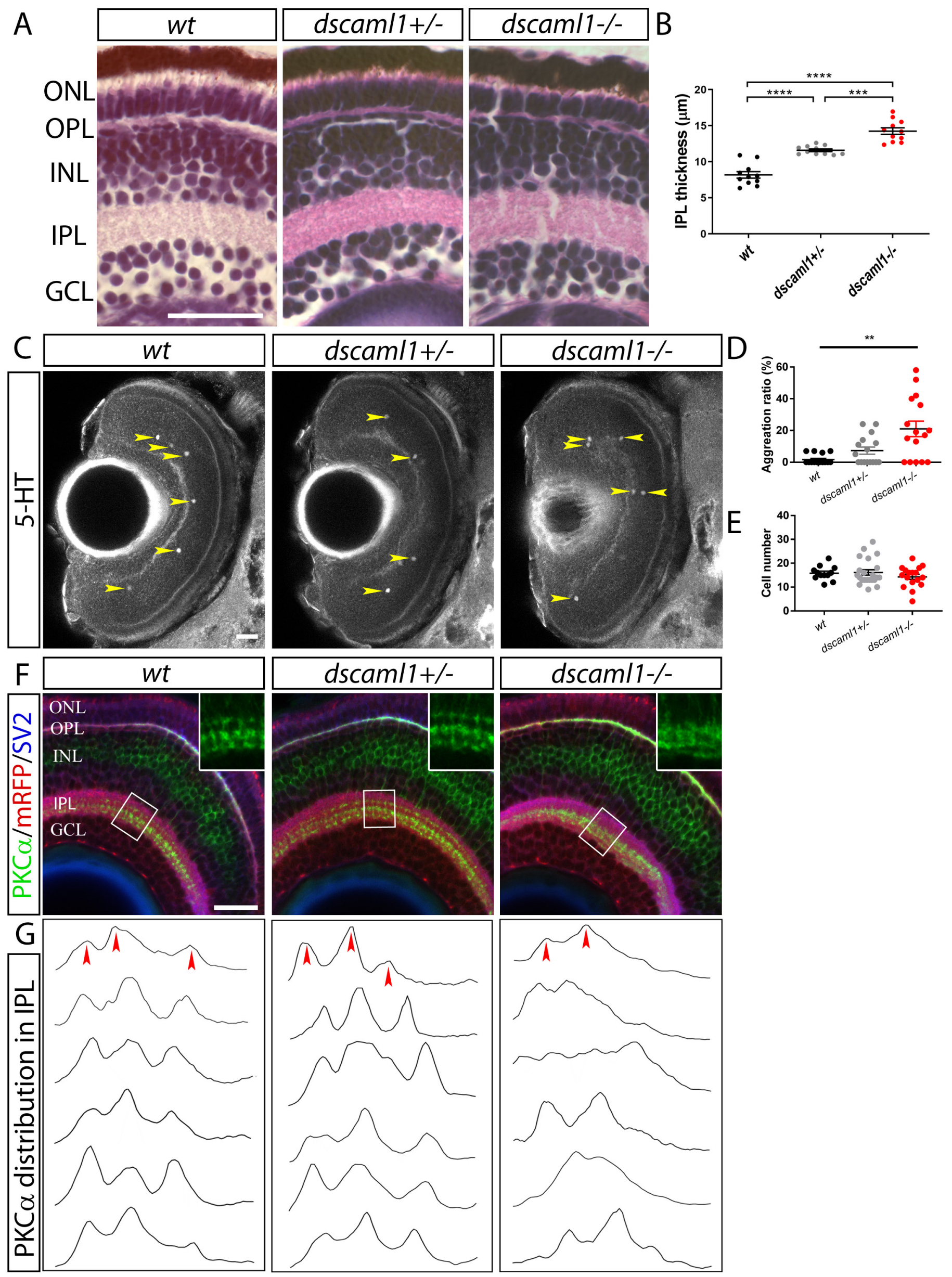

Figure 2 


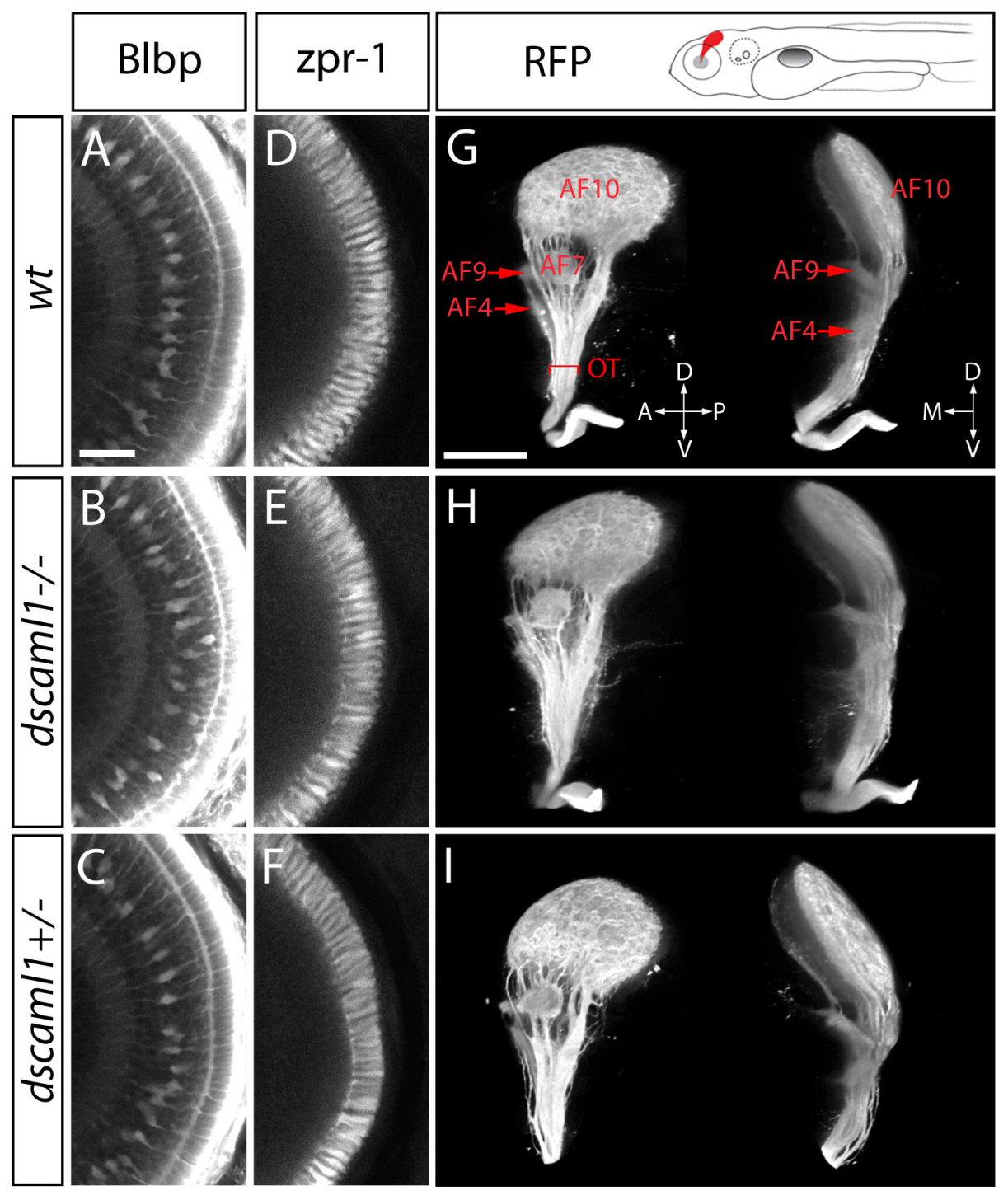

Figure 3 


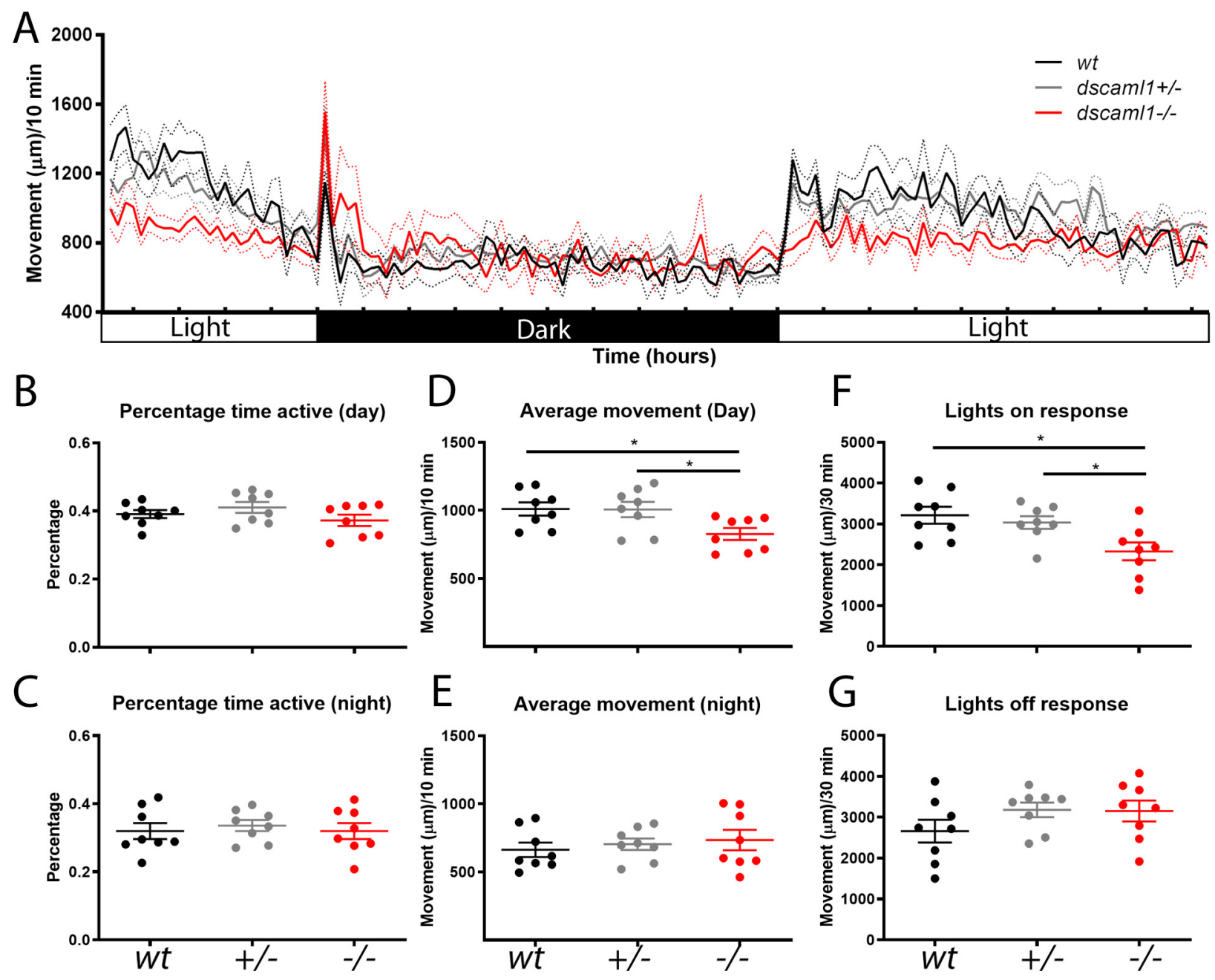

Figure 4 

aCC-BY-NC-ND 4.0 International license.

A

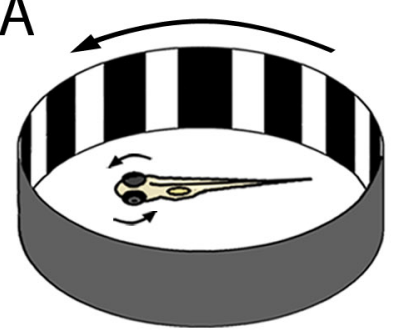

B

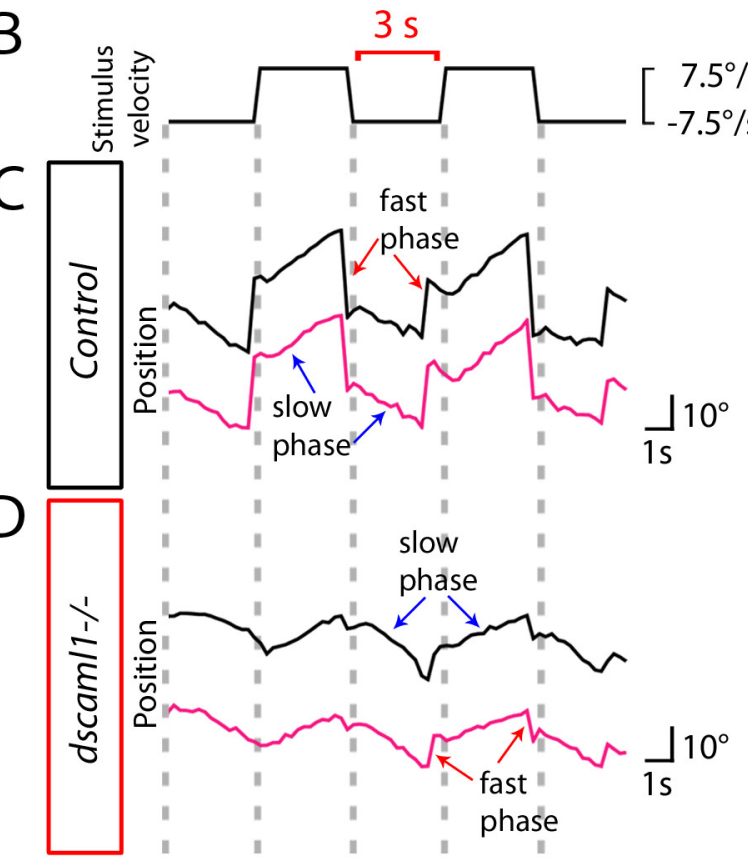

$\mathrm{E}$

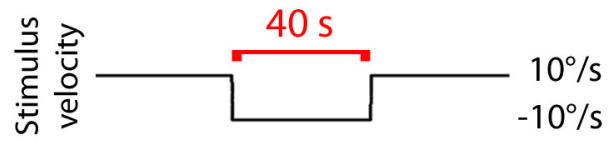

F

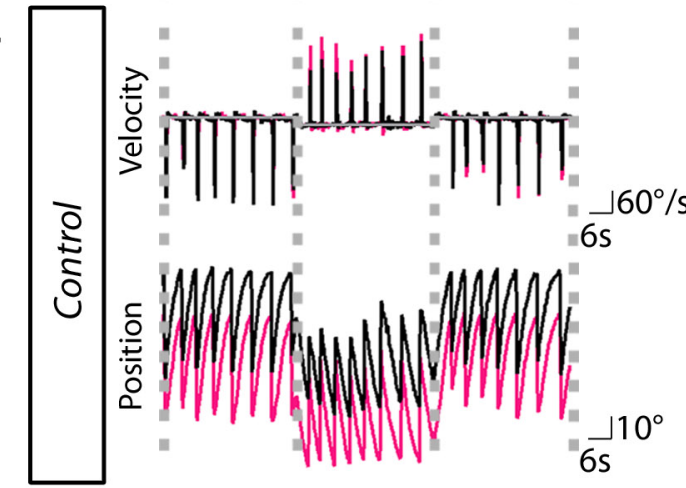

G

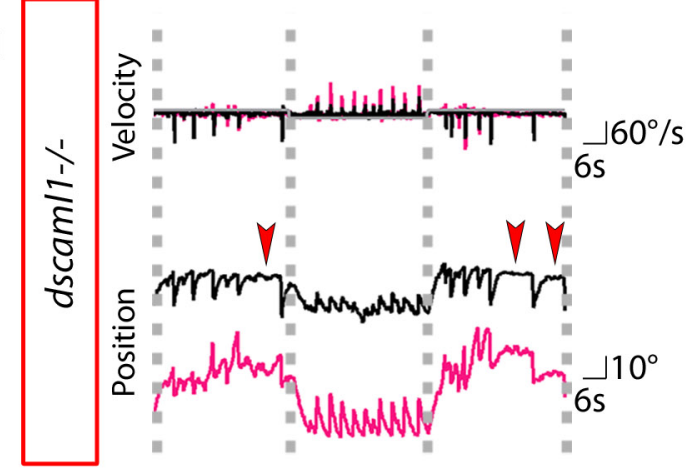

slow fast

phase phase

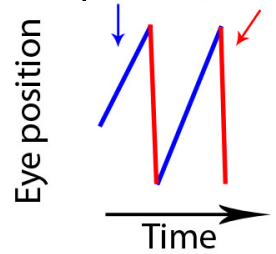

$7.5 \%$<smiles>CCOC</smiles>

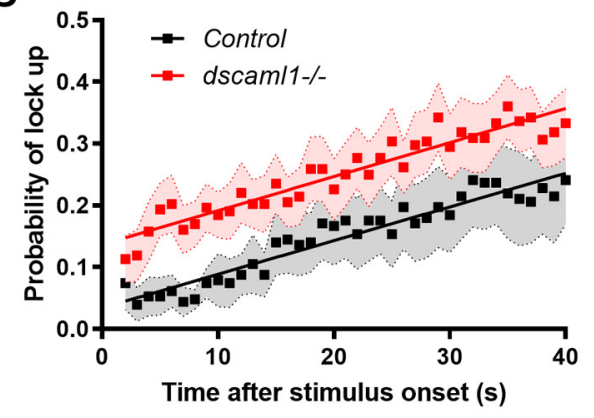

K

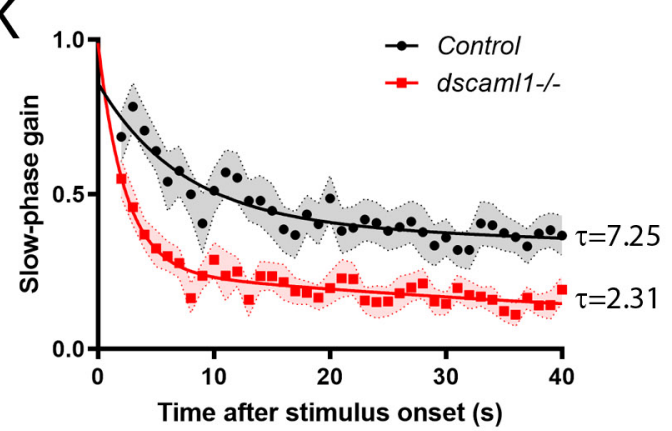

$\mathrm{L}$

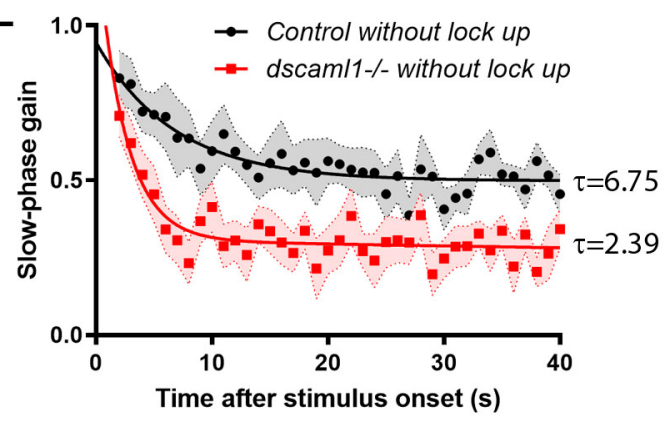

M

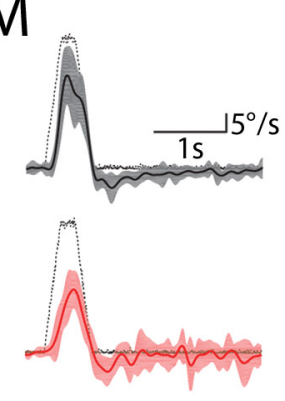

N

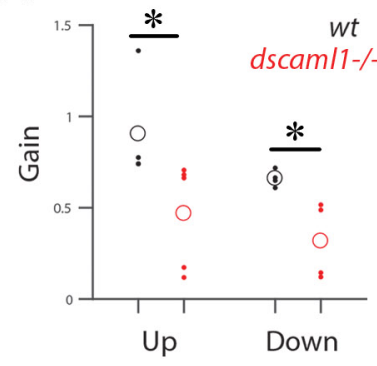


A

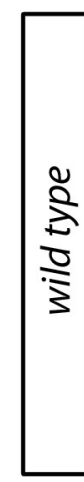<smiles>CC(C)C(C)C(C)C(C)C(C)C(C)C</smiles>

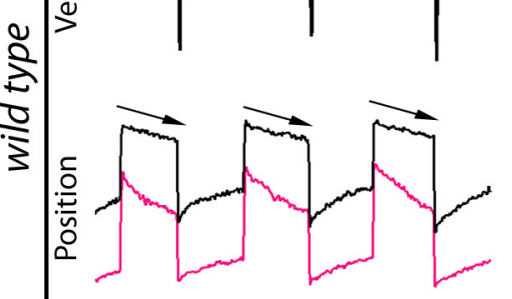
$-160^{\circ}$ \lrcorner $10^{\circ}$ $4 \mathrm{~s}$

B

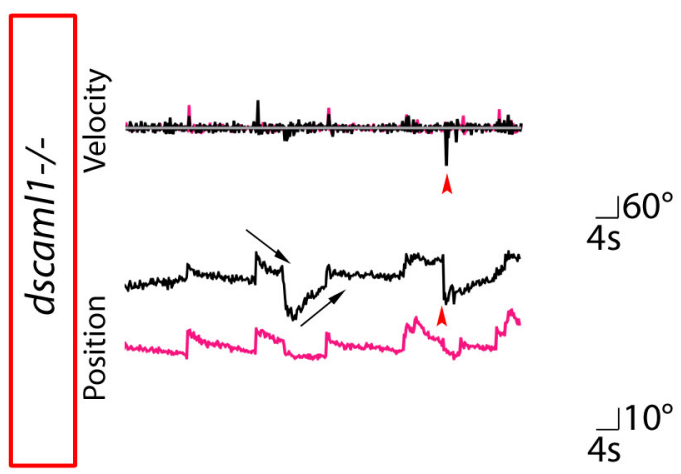

C
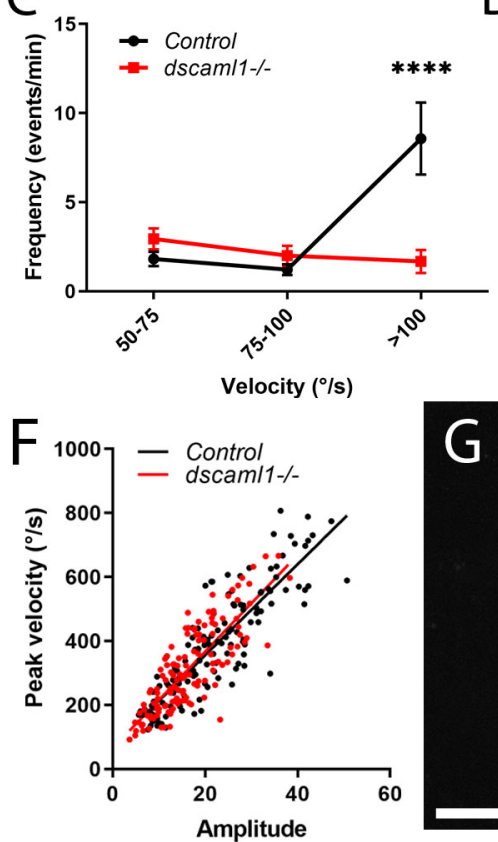

D

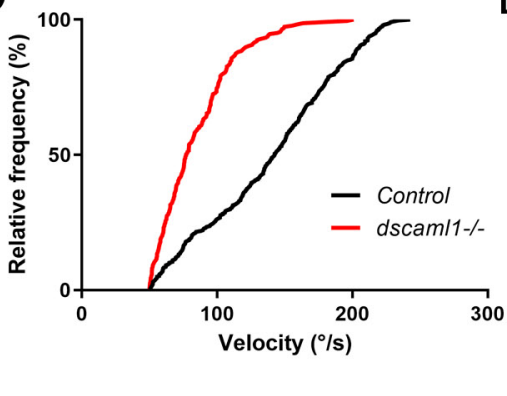

E

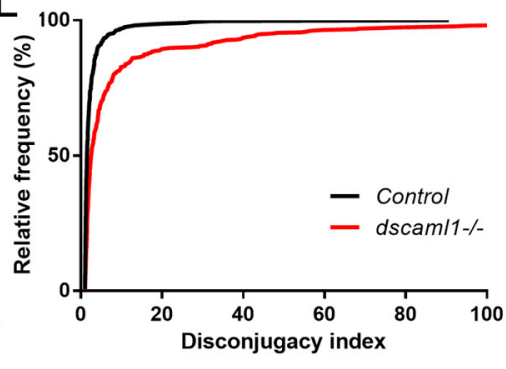

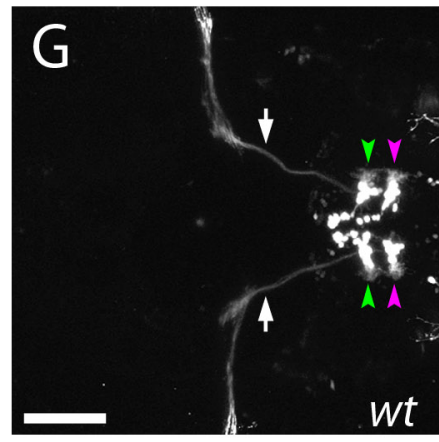
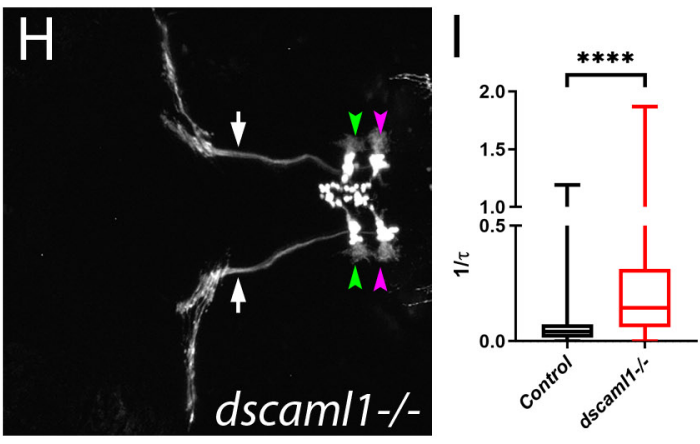

Figure 6 
bioRxiv preprint doi: https://doi.org/10.1101/658161; this version posted June 3, 2019. The copyright holder for this preprint (which was not certified by peer review) is the author/funder, who has granted bioRxiv a license to display the preprint in perpetuity. It is made available under aCC-BY-NC-ND 4.0 International license.

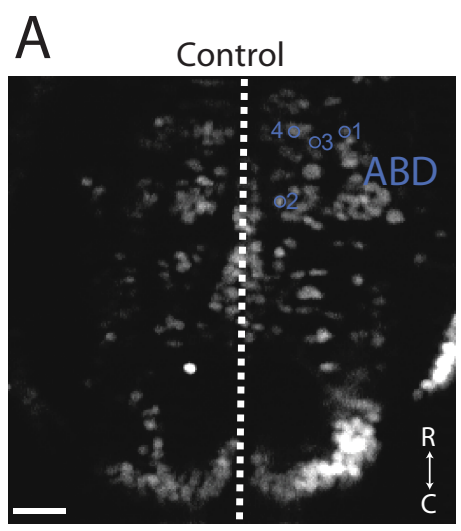

midline

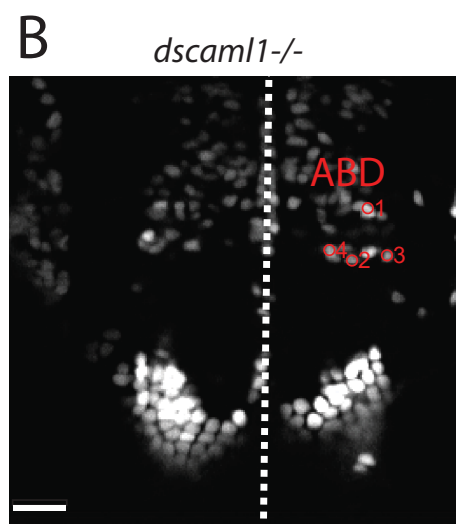

C

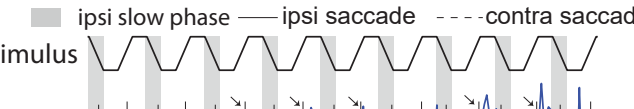

\section{$\mathrm{E}$}
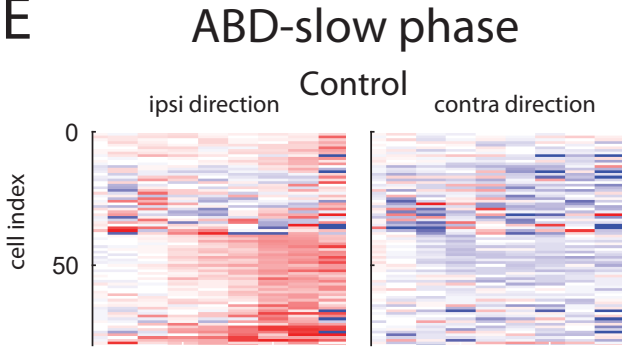

dscam/1-/-

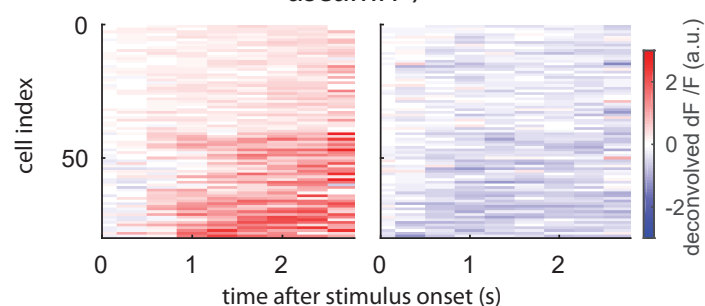

F

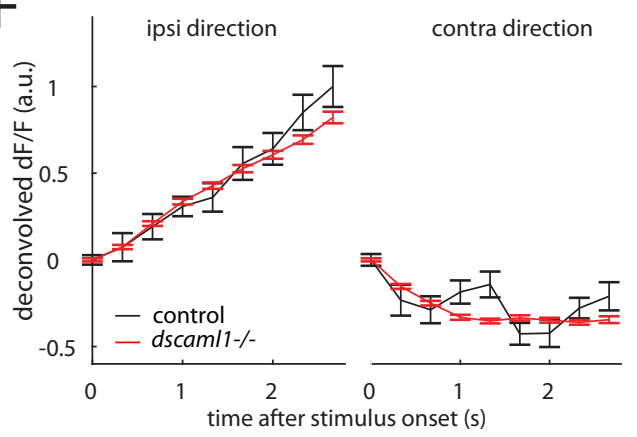

G $\quad$ ABD-saccade
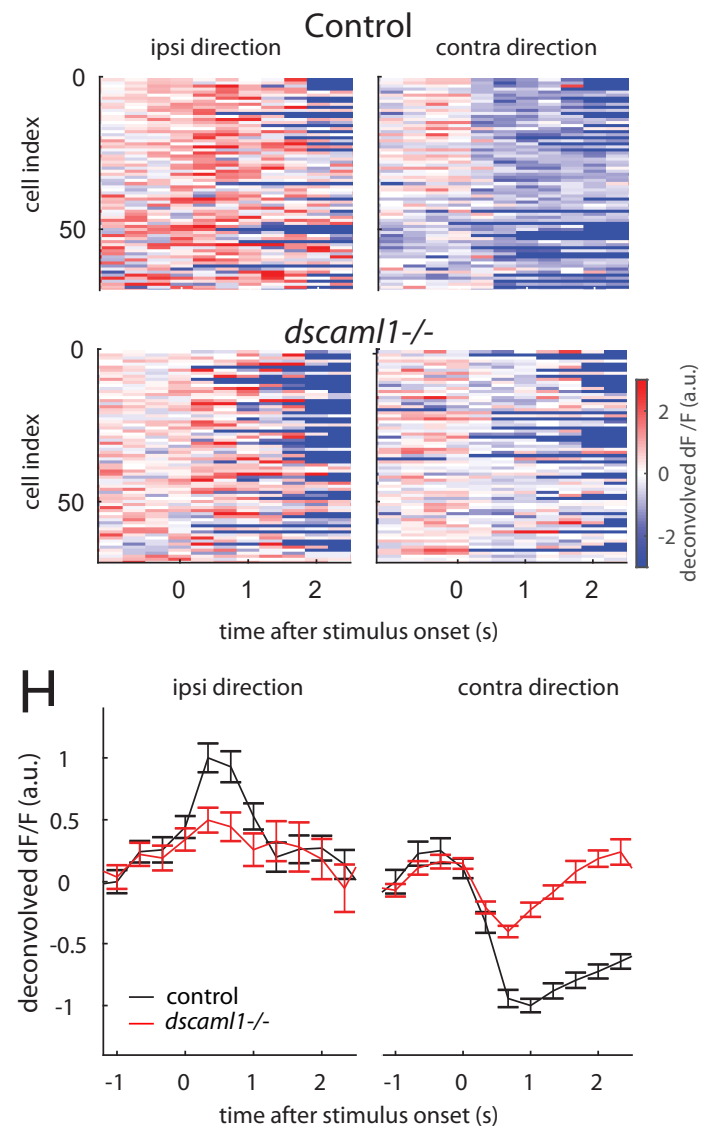

Figure 7 

aCC-BY-NC-ND 4.0 International license.
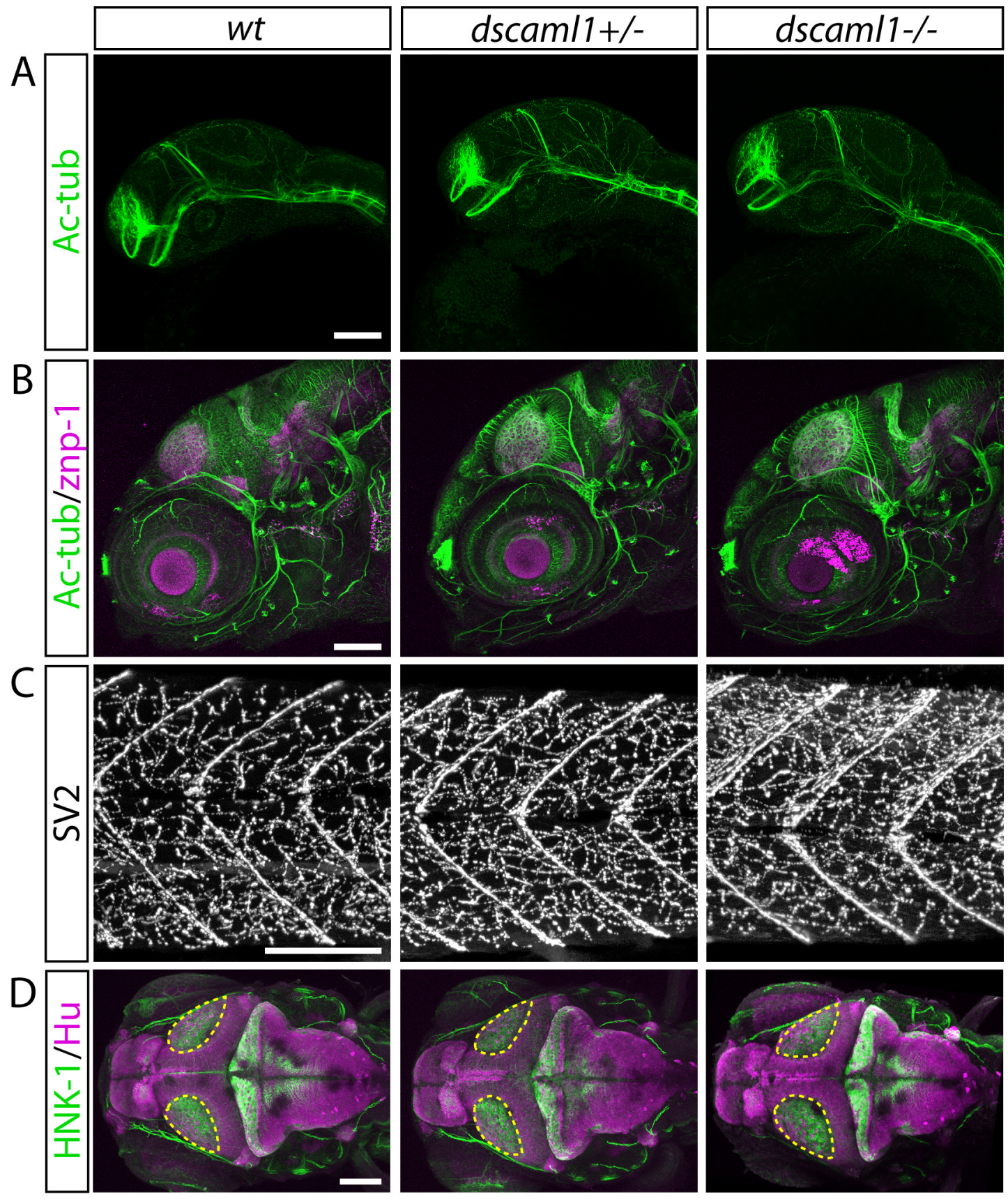

\section{Supplementary Figure S1}


bioRxiv preprint doi: https://doi.org/10.1101/658161; this version posted June 3, 2019. The copyright holder for this preprint (which was not certified by peer review) is the author/funder, who has granted bioRxiv a license to display the preprint in perpetuity. It is made available under aCC-BY-NC-ND 4.0 International license.

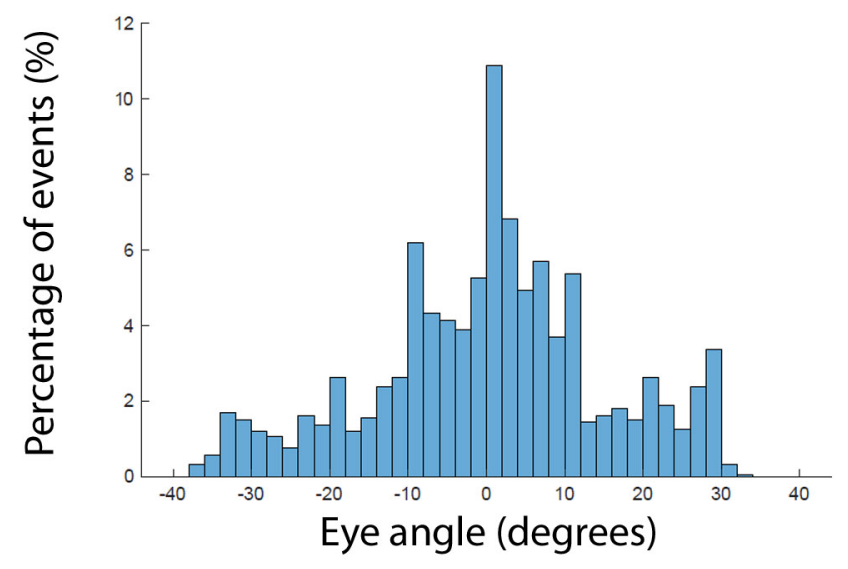

\section{Supplementary Figure S2}




\begin{tabular}{|c|c|c|}
\hline With lock up & Control & dscaml1-/- \\
\hline $\begin{array}{l}Y_{0} \\
\text { Contribution of long } \\
\text { component }\end{array}$ & 47.31 & 25.87 \\
\hline Tau (short) & 7.25 & 2.31 \\
\hline Tau (long) & 305.00 & 72.01 \\
\hline Without lock up & Control & dscaml1-/- \\
\hline $\begin{array}{l}Y_{0} \\
\text { Contribution of long } \\
\text { component }\end{array}$ & 0.9461 & 1.297 \\
\hline Tau (short) & 6.749 & 2.385 \\
\hline Tau (long) & $2.89 \mathrm{E}+08$ & 472.4 \\
\hline
\end{tabular}

Table I. Double exponential decay fit for slow phase gain. 